\title{
Energy-Preserving Time-Varying Schroeder Allpass Filters and Multichannel Extensions
}

\author{
KURT JAMES WERNER,${ }^{*}$ AES Member, FRANÇOIS G. GERMAIN, AND CORY S. GOLDSMITH \\ (kwerner@izotope.com) \\ (fgermain@izotope.com) \\ (cgoldsmith@izotope.com)
}

iZotope, Inc., Cambridge, Massachusetts

\begin{abstract}
We propose time-varying Schroeder allpass filters and Gerzon allpass reverberators that remain energy preserving irrespective of arbitrary variation of their allpass gains or feedback matrices over time. We propose various ways of realizing the unitary matrix involved in the Schroeder structure, based on classic ladder and lattice filters and their generalizations. We show how to construct more elaborate structures including nestings and cascade, giving various strategies for reducing their implementation cost. Extending these algorithms to the multi-input, multi-output case yields time-varying, energy-preserving generalizations of Gerzon's reverberator, providing a link between Schroeder allpass filters and Schelcht's recently proposed "Allpass Feedback Delay Networks." Stability proofs are given for common uses of Schroeder allpass filters, such as inside of Feedback Delay Network reference structures. Finally we give a substantial review of the properties of time-invariant Schroeder allpass filters.
\end{abstract}

\section{INTRODUCTION}

Manfred Schroeder's work on artificial reverb in the 1960s [1, 2] introduced the "Schroeder" (or "comb") allpass filter: a high-order, low-complexity allpass filter characterized by the length of its single delay line and gain coefficient. They can be implemented in various ways: Fig. 1(a) shows the original form [1] and Fig. 1(c) and 1(d) show the two most commonly seen (e.g., [3, 4]). Schroeder allpass filters are also "nested" in cascade with delay lines inside of Feedback Delay Networks (FDNs) [5-10] or another Schroeder allpass [2, 11-16]. Time-varying first-order allpass filters (Schroeder allpasses with length-one delay lines) have also been explored in digital audio effect and synthesizer design [10, 17-25].

Although reverb algorithms are almost always designed from a linear time-invariant (LTI) prototype, it is common to vary gains over time to break up resonances $[5,8,11$, 26-30]. It is essential in varying these gains that the structure's stability be preserved, which can be accomplished by preserving the signal energy during variation. Unfortunately the standard Schroeder allpass filter has been shown not to preserve energy as its coefficient is changed [8].

*Correspondence should be addressed to Kurt James Werner; e-mail: kwerner@izotope.com
In this article we address this issue, introducing a novel family of Schroeder-style allpass filters that are energypreserving during arbitrary and continuous change of their gain coefficient. Preliminary results were given in [31].

Michael Gerzon generalized Schroeder allpass filters to the multi-input multi-output (MIMO) case [9, 32, 33]. His basic design, shown in Fig. 1(b), replaced each of the gains in Schroeder's original (cf., Fig. 1(a)) with a diagonal matrix of identical gains and the delay line with a parallel arrangement of delay lines or a more elaborate unitary system. Just like the single-input single-output (SISO) Schroeder allpass filters from which they are derived, Gerzon-style reverberators do not, in general, preserve energy during coefficient modulation.

In this article we extend the approach of [31], allowing MIMO Gerzon-style reverberators to be created, which are energy-preserving during arbitrary and continuous change of their matrix gains. At the same time we propose some extensions allowing for a wider class of feedback gain matrices. Since these MIMO structures are allpass by construction, they can be considered an alternative, constructive path to the "Allpass FDNs" proposed by Schlecht [34].

Recent research extended Schroeder allpass filters and reverberators, e.g., allowing frequency-dependent gains in Schroeder allpass filters [35]; connecting FDNs to room geometry [36]; adding controls of directional distribution of sound to FDNs [37]; imbuing FDNs with the allpass prop- 


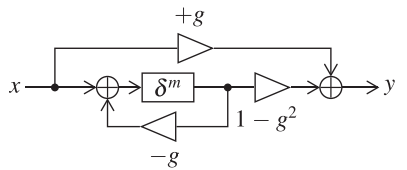

(a) Original Schroeder allpass.

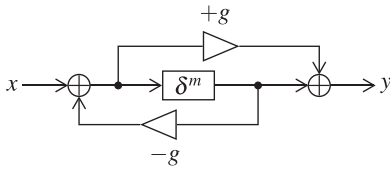

(c) Common Schroeder.

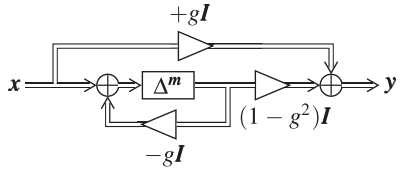

(b) Gerzon's reverberator.

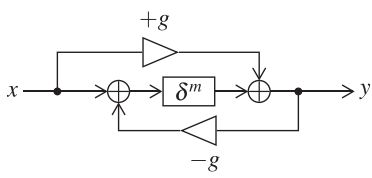

(d) Less common Schroeder.
Fig. 1. Classic Schroeder allpasses (and Gerzon reverb), with input $x(\boldsymbol{x})$, output $y(\boldsymbol{y})$, delay lines $\delta^{m}\left(\Delta^{m}\right)$, and gains parameterized by $g(g \boldsymbol{I})$.

erty [34]; generalizing FDN feedback to a matrix of filters [38], including the case of velvet noise [39, 40] feedback matrices in particular [41]; and studying coupled and parallel FDNs [42, 43]. This article complements these works, providing new insight on Schroeder allpass filters and FDN architectures with good time-varying properties.

The rest of the article is structured as follows. We start with preliminaries (SEC. 1). In a first part we present our proposed approach to building energy-preserving, timevarying Schroeder allpass filters (SEC. 2), including their building blocks, algorithm recipe, and more advanced combinations, including cascades and nestings. Next we discuss techniques for saving multipliers in groups of Schroeder allpass filters (SEC. 3) and a case study demonstrating some of the proposed filters (SEC. 4). In a second part we present a MIMO generalization allowing the creation of energy-preserving time-varying "Gerzon-style" reverberation algorithms (SEC. 5), again giving case studies (SEC. 6) on how the proposed techniques improve existing MIMO structures. SEC. 7 concludes and an APPENDIX thoroughly reviews various properties of the LTI Schroeder allpass filter.

\section{PRELIMINARIES}

Here we review important concepts such as the sign convention (SEC. 1.1), our time indices and delay notation (SEC. 1.2), L2 and matrix-norm and unitary (SEC. 1.3) and paraunitary matrices (SEC. 1.4), energy-preserving systems (SEC. $1.5)$, and a state space variation called "delay state space" (SEC. 1.6).

\subsection{Sign Convention}

The classic Schroeder allpass filter [1] (Fig. 1) has a feedforward gain $-g$ and feedback gain $+g$; we call this the "negative sign convention." However many sources use the opposite "positive sign convention": feedforward gain $+g$ and feedback gain $-g$. In rare cases both sign conventions are intermingled (e.g., [44]). One can see evidence of the variety of conventions later in the article (Table 4).

In this article we use the "positive sign convention" due to its use in recent extensions to Schroeder allpass filters [31,
35], out of deference to the historical usage at CCRMA [45], and because it is congruent with standard transfer function conventions. Adherents to the other sign convention may simply substitute $-g$ for $g$ throughout.

\subsection{Time Indices}

This article is primarily concerned with time-varying allpass structures. So, nearly every gain and signal has a time index $[n]$. The main exception to this is the discussion in the APPENDIX of the basic LTI Schroeder allpass filters, where, since the $z$-transform is used to discuss the transfer function, the gain cannot vary over time. For compactness we suppress the notation of the time index except in places where multiple time indices appear in the same expression, e.g., in difference equations or summations over time indices. In these instances it will be notated with square brackets, e.g., input $x[n]$, output $y[n]$, allpass gain $g[n]$. We also use an operator $\delta^{1}$ to represent a one-sample delay. A length- $M$ delay line can be formed by $\delta^{M}=\prod_{m=0}^{M-1} \delta^{1}$.

\subsection{Norms and Unitary Matrices}

This article relies on certain properties of the L2- and matrix-norms and unitary matrices, which we review here. We will present many of these theoretical aspects with complex matrices even though practically we mainly only care about the real-valued cases. We do this to be consistent with prior artificial reverb literature and in case the complex case somehow becomes useful in the future.

The L2-norm of a length- $M$ vector $\boldsymbol{x} \in \mathbb{C}^{M \times 1}$ is

$$
\|\boldsymbol{x}[n]\|=\left(\sum_{m=0}^{M-1}\left|x_{m}[n]\right|^{2}\right)^{1 / 2},
$$

where $x_{m}[n]$ is the $m$ th entry of $\boldsymbol{x}$ at time $[n]$. We use the L2norm of scalar quantities over time (square root of signal energy). For scalar $x[n],\|x[\cdot]\|$ is defined as

$$
\|x[\cdot]\|=\left(\sum_{n=-\infty}^{\infty}|x[n]|^{2}\right)^{1 / 2} .
$$

This is distinguished from the L2-norm over entries by the inclusion of the bracketed dot.

The matrix norm $\|\boldsymbol{M}\|$ of a matrix $\boldsymbol{M}$ is defined as the largest singular value of $\boldsymbol{M}$. A matrix $\boldsymbol{U} \in \mathbb{C}^{M \times M}$ is called unitary when it satisfies

$$
\boldsymbol{U} \boldsymbol{U}^{\mathrm{H}}=\boldsymbol{U}^{\mathrm{H}} \boldsymbol{U}=\boldsymbol{I},
$$

where $(\cdot)^{\mathrm{H}}$ is the Hermitian conjugate, the transpose and conjugate of the coefficients and $\boldsymbol{I}$ is the $M \times M$ identity matrix. Throughout this article identity and zero (0) matrices are assumed to have the appropriate implied dimensions. An important property of unitary matrices is that they preserve the L2-norm during vector multiplication

$$
\boldsymbol{y}=\boldsymbol{U} \boldsymbol{x} \quad \Longrightarrow \quad\|\boldsymbol{y}[n]\|=\|\boldsymbol{x}[n]\|,
$$

for any $\boldsymbol{x}, \boldsymbol{y} \in \mathbb{C}^{M \times 1}$.

Throughout this article we often refer to a specific unitary matrix, which can be identified by the presence of an 
argument in parentheses. This is the unitary dilation of the contraction $\boldsymbol{G} \in \mathbb{C}^{N \times N}$ on $\mathbb{C}^{2 N \times 2 N}$ as [46, pp. 13-19]:

$$
\boldsymbol{U}(\boldsymbol{G})=\left[\begin{array}{cc}
-\boldsymbol{G} & \mathrm{D}_{\boldsymbol{G}^{\mathrm{H}}} \\
\mathrm{D}_{\boldsymbol{G}} & \boldsymbol{G}^{\mathrm{H}}
\end{array}\right]
$$

with $\boldsymbol{U}(\boldsymbol{G}) \in \mathbb{C}^{2 M \times 2 M}$. We assume $\|\boldsymbol{G}\|<1$, i.e., that $\boldsymbol{G}$ is contractive, and use the compact notation

$$
\mathrm{D}_{\Gamma}=\left(\boldsymbol{I}-\Gamma^{\mathrm{H}} \Gamma\right)^{1 / 2},
$$

where $\mathrm{D}_{(\text {. . }}$ is the "defect operator."

In the scalar case used for constructing SISO allpass filters we have $\boldsymbol{U}(g) \in \mathbb{C}^{2 \times 2}$, where $|g|<1$, defined by

$$
\boldsymbol{U}(g)=\left[\begin{array}{cc}
-g & \mathrm{D}_{\bar{g}} \\
\mathrm{D}_{g} & \bar{g}
\end{array}\right]
$$

with scalar defect operator

$$
\mathrm{D}_{\gamma}=\sqrt{1-\gamma^{2}}
$$

Throughout this article we will often say that a matrix $\boldsymbol{M}$ is diagonally similar to a unitary matrix $\boldsymbol{U}$. By definition $\boldsymbol{M}$ is diagonally similar to $\boldsymbol{U}$ if there exists a matrix $\underline{\boldsymbol{P}}=$ $\operatorname{diag}(\boldsymbol{p}), \boldsymbol{p} \in \mathbb{R}^{M \times 1}$ such that $\boldsymbol{M}$ can be written as

$$
\boldsymbol{M}=\underline{\boldsymbol{P}}^{-1} \boldsymbol{U} \underline{\boldsymbol{P}} .
$$

We use a line under a matrix as a reminder that the matrix is diagonal $\left(\underline{\boldsymbol{M}}_{i, j}=0, \forall i \neq j\right)$.

\subsection{Paraunitary Matrices}

Unitarity is only defined for scalar matrices. However we often have to consider matrices that involve filters, e.g., a diagonal matrix of delay lines. So it is useful to consider paraunitarity. A matrix $\boldsymbol{U}(z)$ is called paraunitary when

$$
\boldsymbol{U}(z) \boldsymbol{U}^{\mathrm{H}}\left(z^{-1}\right)=\boldsymbol{I} .
$$

Note well that $\boldsymbol{U}^{\mathrm{H}}\left(z^{-1}\right)$ has transpose-conjugate coefficients w.r.t. $\boldsymbol{U}(z)$ and inverse powers of $z$ (rather than conjugated) $[10,47,48]$. If a matrix is unitary on the unit circle, $\boldsymbol{U}\left(\mathrm{e}^{\mathrm{j} \omega}\right) \boldsymbol{U}^{\mathrm{H}}\left(\mathrm{e}^{-\mathrm{j} \omega}\right)=\boldsymbol{I}$, then it is also paraunitary [47, 48].

Paraunitary systems can be combined in several ways to create new paraunitary systems $[9,49]$ (we suppress the $(z)$ argument in the rest of this section for compactness). We mention three known elementary strategies [9]:

1. Two paraunitary matrices $\boldsymbol{U}, \boldsymbol{V} \in \mathbb{C}^{M \times M}$ of the same size can be put in cascade to create a new paraunitary matrix $\boldsymbol{W} \in \mathbb{C}^{M \times M}$, as shown in Fig. 2(a) and defined by

$$
\boldsymbol{W}=\boldsymbol{V} \cdot \boldsymbol{U}
$$

2. Two paraunitary matrices $\boldsymbol{U} \in \mathbb{C}^{M \times M}$ and $\boldsymbol{V} \in$ $\mathbb{C}^{N \times N}$, which may be different sizes, can be put in parallel to create a larger paraunitary matrix $\boldsymbol{W} \in \mathbb{C}^{(M+N) \times(M+N)}$, as shown in Fig. 2(b). They

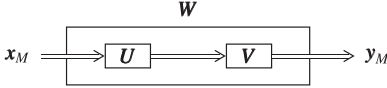

(a) Cascade

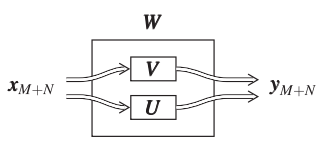

(b) Parallel.

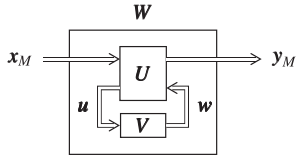

(c) Nested.
Fig. 2. Three ways of creating a new energy-preserving structures from two other energy-preserving structures.

become block diagonal entries of the new matrix; it is their "direct sum" $(\oplus)$ :

$$
\boldsymbol{W}=\boldsymbol{U} \oplus \boldsymbol{V}=\operatorname{diag}(\boldsymbol{U}, \boldsymbol{V})=\left[\begin{array}{ll}
\boldsymbol{U} & \mathbf{0} \\
\mathbf{0} & \boldsymbol{V}
\end{array}\right]
$$

3. Two paraunitary matrices $\boldsymbol{U} \in \mathbb{C}^{(M+N) \times(M+N)}$ and $\boldsymbol{V} \in \mathbb{C}^{N \times N}$ can be combined in a feedback arrangement to create a new paraunitary matrix $\boldsymbol{W} \in \mathbb{C}^{M \times M}$, as shown in Fig. 2(c). Defining the partitions of $\boldsymbol{U}$ by

$$
\boldsymbol{U}=\left[\begin{array}{ll}
\boldsymbol{U}_{M, M} & \boldsymbol{U}_{M, N} \\
\boldsymbol{U}_{N, M} & \boldsymbol{U}_{N, N}
\end{array}\right],
$$

where the partitions each have size $\boldsymbol{U}_{\phi, \psi} \in \mathbb{C}^{\phi \times \psi}$, the new paraunitary matrix is defined by $F(\boldsymbol{U}, \boldsymbol{V})$, as a function of by $\boldsymbol{U}$ and $\boldsymbol{V}$, by

$$
\begin{aligned}
& \boldsymbol{W}=\mathrm{F}(\boldsymbol{U}, \boldsymbol{V}) \\
& =\boldsymbol{U}_{M, M}+\boldsymbol{U}_{M, N} \boldsymbol{U}_{N, N}\left(\boldsymbol{I}_{N}-\boldsymbol{V} \boldsymbol{U}_{N, N}\right)^{-1} \boldsymbol{U}_{N, M} .
\end{aligned}
$$

This also involves inner signal vectors $\boldsymbol{u}, \boldsymbol{w} \in \mathbb{C}^{N \times 1}$.

\subsection{Energy-Preserving Systems}

More generally energy-preserving time-varying systems (that do not have $z$-transforms) can be combined in the same three ways to produce more complicated energy-preserving time-varying systems. An $M$-input, $M$-output MIMO system is energy-preserving if it satisfies:

$$
\sum_{m=0}^{M-1}\left\|y_{m}[\cdot]\right\|=\sum_{m=0}^{M-1}\left\|x_{m}[\cdot]\right\|
$$

Various systems we have mentioned so far are energypreserving, including any paraunitary system $\boldsymbol{U}(z)$, delay line $\delta^{m}$, and parallel arrangement of $K$ delay lines: $\Delta^{\boldsymbol{m}}=\bigoplus_{k=0}^{K-1} \delta^{m_{k}}, \boldsymbol{m} \in \mathbb{Z}_{+}^{K \times 1}$. Remarkably time-varying unitary matrices $\boldsymbol{U}[n]$ are also energy-preserving, since unitary matrices preserve the L2-norm of a vector. Squaring both sides of (4) and summing over time yields (14), verifying energy preservation.

Two energy-preserving systems in cascade (Fig. 2(a)) or parallel (Fig. 2(b)) yield a new energy-preserving system. Two nested energy-preserving systems (Fig. 2(c)) also 


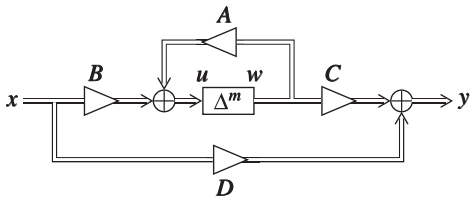

(a) Delay state space.

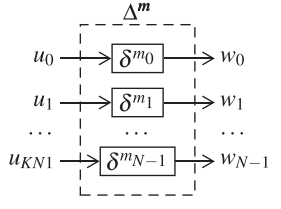

(b) Delay lines detail.
Fig. 3. General delay state space form.

produce a new energy-preserving system, but since it is slightly more complicated we derive it in some detail.

Assume that both $\boldsymbol{U} \in \mathbb{R}^{M \times M}$ and $\boldsymbol{V} \in \mathbb{R}^{L \times L}$ are energypreserving. Given $\boldsymbol{x}, \boldsymbol{y} \in \mathbb{R}^{M \times 1}$ and $\boldsymbol{u}, \boldsymbol{w} \in \mathbb{R}^{L \times L}$, we have

$\sum_{M=0}^{M-1}\left\|x_{m}[\cdot]\right\|+\sum_{\ell=0}^{L-1}\left\|w_{\ell}[\cdot]\right\|=\sum_{M=0}^{M-1}\left\|y_{m}[\cdot]\right\|+\sum_{\ell=0}^{L-1}\left\|u_{\ell}[\cdot]\right\|$

$$
\sum_{\ell=0}^{L-1}\left\|u_{\ell}[\cdot]\right\|=\sum_{\ell=0}^{L-1}\left\|w_{\ell}[\cdot]\right\| .
$$

Subtracting (16) from (15) yields (14), verifying energy preservation.

\subsection{Delay State Space Form}

In this article, to study the stability of certain structures, we rewrite their equations in the so-called "delay statespace" form [34, 50], shown in Fig. 3. For systems that involve long runs of delays we can compactly describe the entire system by

$$
\left[\begin{array}{l}
u \\
y
\end{array}\right]=\underbrace{\left[\begin{array}{ll}
A & B \\
C & D
\end{array}\right]}_{S}\left[\begin{array}{l}
w \\
x
\end{array}\right], \quad w=\Delta^{m} u,
$$

with matrices $\boldsymbol{A} \in \mathbb{R}^{N \times N}, \boldsymbol{B} \in \mathbb{R}^{N \times K}, \boldsymbol{C} \in \mathbb{R}^{K \times N}$, and $\boldsymbol{D} \in \mathbb{R}^{K \times K}$; vectors $\boldsymbol{m}, \boldsymbol{u}, \boldsymbol{w} \in \mathbb{R}^{N \times 1}$ and $\boldsymbol{x}, \boldsymbol{y} \in \mathbb{R}^{K \times 1}$; and system matrix $\boldsymbol{S} \in \mathbb{R}^{(K+N) \times(K+N)}$. When $\boldsymbol{m}=\mathbf{1}$ (a vector of $1 \mathrm{~s}$ ), this is equivalent to standard state space. We will often refer to FDNs with $N$ delay lines as " $N$-FDNs" [51].

\section{PROPOSED SISO STRUCTURES}

In this section we propose (extending [31]) designs for energy-preserving time-varying SISO Schroeder all- pass filters, which improve on the classic structures by being unconditionally stable no matter how their coefficients are varied over time. This approach also yields nested and cascaded structures and forms a foundation for the MIMO structures we treat in SEC. 5. We present the building blocks of these structures (SEC. 2.1), a recipe for combining them into energy-preserving SISO structures (SEC. 2.2), and a discussion of the resulting structures (SEC. 2.3).

\subsection{SISO Building Blocks}

The building blocks of our proposed structures are shown in Fig. 4. The first type of block (Fig. 4(a)) is a lossless twoport, well known from filter design and speech synthesis: Fig. 4(b)-4(j) shows nine versions. Of these nine there are five distinct matrix descriptions, which we call the "type," indicated by a roman numeral I-V. Types I-IV each have two possible realizations; e.g., Type III can be realized in four-multiplier form (Fig. 4(c), the classic Kelly-Lochbaum block [52]) or in one-mult. form (Fig. 4(i)). Type V (the normalized structure, Fig. 4(b)) only has a single realization.

All five of the types (hence, all nine of the realizations) can be described by a single matrix $\boldsymbol{L}(g, \xi)$

$$
\boldsymbol{L}(g, \xi)=\left[\begin{array}{cc}
g & \mathrm{D}_{g} \xi \\
\mathrm{D}_{g} / \xi & -g
\end{array}\right]=\left[\begin{array}{ll}
\ell_{00} & \ell_{01} \\
\ell_{10} & \ell_{11}
\end{array}\right],
$$

where we require $\xi \neq 0$ to bound the multipliers and assume without loss of generality that $\xi>0$. Recall that the scalar defect operator $\mathrm{D}_{g}$ is defined in (8). The correspondence between building blocks, their types, the entries of $\boldsymbol{L}(g, \xi)$, and the value of $\xi$ to represent each structure with the generalized structure are shown in Table 1.

$\boldsymbol{L}(g, \xi)$ is unilossless [53] $\forall \xi \neq 0$ since it is diagonally similar to $\boldsymbol{U}(g)$ by $\boldsymbol{P}=\operatorname{diag}(1,1 / \xi)$. However it is only unitary for $\xi=1$. Hence all of the two-ports shown, or any version of Fig. 4(a) with other values of $\xi$, are unilossless. Only the normalized structure (Type V, Fig. 4(b)) is unitary.

The next type of building block is the "transformer," shown in Fig. 4(k). The appropriate transformer value $\xi$ for each type is simply the same value that parameterizes the general block. Transformers will always be shown as shaded, purely for visual ease.

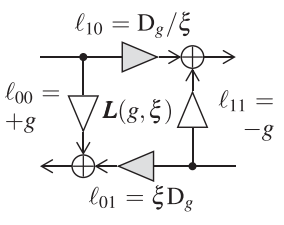

(a) generalized.

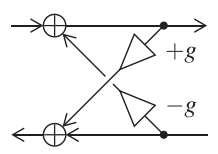

(g) 2-mult., I.

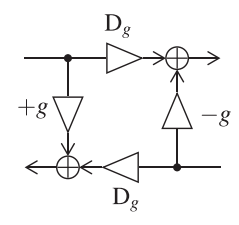

(b) norm., V.

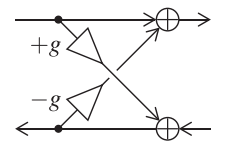

(h) 2-mult. ${ }^{\top}$, II.

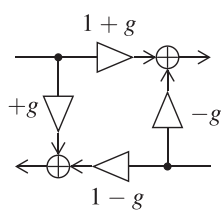

(c) 4-mult., III.

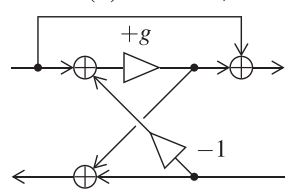

(i) 1-mult., III.

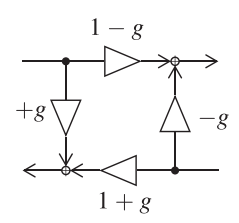

(d) 4-mult. ${ }^{\top}$, IV.

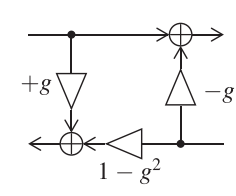

(e) 3-mult., I.

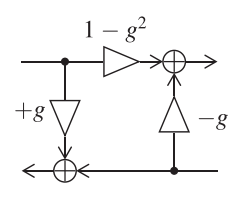

(f) 3-mult. ${ }^{\top}$ II.

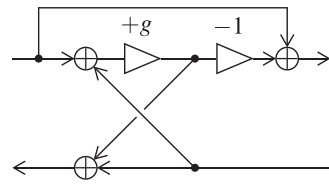

(j) 1-mult. ${ }^{\top}$, IV.

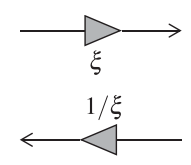

(k) transformer.

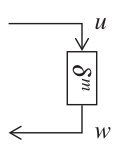

(1) delays

Fig. 4. Building blocks of the proposed algorithm, including ladder/lattice two-ports, transformer, and delay line. 
Table 1. Two-port entries, corresponding transformer gains, and difference equation coefficients for unnormalized structures.

\begin{tabular}{|c|c|c|c|c|c|c|c|c|c|c|}
\hline \multirow[b]{2}{*}{ Name } & \multirow[b]{2}{*}{ Type } & \multicolumn{4}{|c|}{ Two-port $\boldsymbol{L}(g, \xi)$ entries } & \multicolumn{2}{|c|}{ Transformer } & \multicolumn{3}{|c|}{ Un-corrected difference eqn. coefficients } \\
\hline & & $\ell_{00}$ & $\ell_{01}$ & $\ell_{10}$ & $\ell_{11}$ & $\xi$ & $1 / \xi$ & $b_{0, n}$ & $b_{M, n}$ & $a_{M, n}$ \\
\hline 1- \& 4-mult. & III & $+g$ & $1-g$ & $1+g$ & $-g$ & $\sqrt{\frac{1-g}{1+g}}$ & $\sqrt{\frac{1+g}{1-g}}$ & $+g[n]$ & $\frac{1-g[n]}{1-g[n-M]}$ & $g[n-M] \frac{1-g[n]}{1-g[n-M]}$ \\
\hline 1- \& 4-mult. ${ }^{\top}$ & IV & $+g$ & $1+g$ & $1-g$ & $-g$ & $\sqrt{\frac{1+g}{1-g}}$ & $\sqrt{\frac{1-g}{1+g}}$ & $+g[n]$ & $\frac{1+g[n]}{1+g[n-M]}$ & $g[n-M] \frac{1+g[n]}{1+g[n-M]}$ \\
\hline 2- \& 3-mult. & I & $+g$ & $1-g^{2}$ & 1 & $-g$ & $\mathrm{D}_{g}$ & $1 / \mathrm{D}_{g}$ & $+g[n]$ & $\frac{1-g[n]^{2}}{1-g[n-M]^{2}}$ & $g[n-M] \frac{1-g[n]^{2}}{1-g[n-M]^{2}}$ \\
\hline 2- \& 3-mult. ${ }^{\top}$ & II & $+g$ & 1 & $1-g^{2}$ & $-g$ & $1 / \mathrm{D}_{g}$ & $\mathrm{D}_{g}$ & $+g[n]$ & 1 & $g[n-M]$ \\
\hline Normalized & $\mathrm{V}$ & $+g$ & $\mathrm{D}_{g}$ & $\mathrm{D}_{g}$ & $-g$ & 1 & 1 & $+g[n]$ & $\sqrt{\frac{1-g[n]^{2}}{1-g[n-M]^{2}}}$ & $g[n-M] \sqrt{\frac{1-g[n]^{2}}{1-g[n-M]}}$ \\
\hline
\end{tabular}

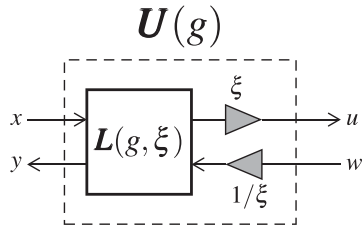

(a) Transformer "inside."

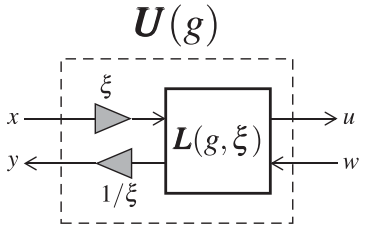

(b) Transformer "outside."
Fig. 5. Two ways of forming a two-port unitary matrix $\boldsymbol{U}(g)$ from two-port $\boldsymbol{L}(g, \xi)$ and a transformer.

The two ways of forming an orthogonal block from a transformer and a ladder/lattice block are shown in Fig. 5. While it is evident by inspection that that two structures are equivalent, a proof is given in [31].

The last type of building block is a length- $m$ delay line: $\delta^{m}$. Recall that delay lines are paraunitary and hence energypreserving. In closing we also mention that a sign flip can be inserted on the output of the transformer or delay line without affecting the energy preservation.

\subsection{SISO Recipe}

We have now assembled a kit of basic energy-preserving building blocks: delay lines and various realizations of unitary blocks $\boldsymbol{U}(g)$. Now we can use the rules in SEC. 1.5 to combine these building blocks into time-varying energypreserving Schroeder allpass filters and more complicated related SISO structures.

To produce a SISO energy-preserving Schroeder allpass filter we simply terminate one of the blocks $\boldsymbol{U}(\mathrm{g})$ (comprising a normalized block or a transformer and a lossless ladder/lattice block in either order) on delay line. Using the notation of SEC. 1.4, this structure is defined by $\mathrm{F}\left(\boldsymbol{U}(g), \delta^{m}\right)$ and shown in Fig. 6(a). All possible realizations of this structure have the difference equation

$$
\begin{aligned}
& y[n]=g[n] x[n] \\
& \quad+\frac{\mathrm{D}_{g[n]}}{\mathrm{D}_{g[n-M]}}(x[n-M]-g[n-M] y[n-M]) .
\end{aligned}
$$

On the right side of Table 1 the difference equation coefficients for the non-stabilized structures that result from omitting the transformer are shown-notice that they are quite different. As an example, realizing $\boldsymbol{U}(g)$ as a 2-mult. (Type I, Fig. 4(g)) with the transformer "outside" gives the signal flow graph shown in Fig. 7(a).

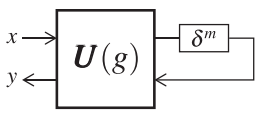

(a) Single.

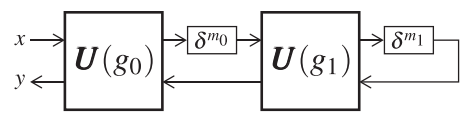

(b) Nested

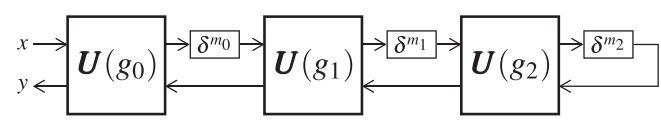

(c) Doubly nested

Fig. 6. Three examples of forming energy-preserving Schroeder allpass filters from the building blocks.

Using the nine different realizations of $\boldsymbol{L}(g, \xi)$ and the two possible transformer locations leads to the 17 different new structures shown in Fig. 8. The normalized (Type V) structure has no distinction between the transformer location because in that case $\xi=1 / \xi=1$. All of these structures are energy-preserving under time-varying $g$.

The same approach can also be used to create more elaborate structures. For instance, replacing the delay line with the cascade of a delay line and another Schroeder allpass filter, $\mathrm{F}\left(\boldsymbol{U}\left(g_{0}\right), \mathrm{F}\left(\boldsymbol{U}\left(g_{1}\right), \delta^{m_{1}}\right) \delta^{m_{0}}\right)$, as shown in Fig. 6(b), yields an energy-preserving nested Schroeder allpass filter, shown in Fig. 7(b). Continuing the process further by $\mathrm{F}\left(\boldsymbol{U}\left(g_{0}\right), \mathrm{F}\left(\boldsymbol{U}\left(g_{1}\right), \mathrm{F}\left(\boldsymbol{U}\left(g_{2}\right), \delta^{m_{2}}\right) \delta^{m_{1}}\right) \delta^{m_{0}}\right)$, as shown in Fig. 6(c), yields an energy-preserving doubly nested Schroeder allpass filter, shown in Fig. 7(c). In these examples we have continued illustrating the unitary matrices with two-mult. (Type I, Fig. 4(g)) blocks but emphasize that any of the 17 proposed structures may be used instead.

\subsection{SISO Structures Discussion}

None of the 17 structures shown in Fig. 8 appear to have been reported before. The implementation costs of each filter structure - in terms of multiplies $(\times)$, adds $(+)$, sign inversions (inv.), and delay registers $(\delta)$ - are shown in Table 2. The two-multiplier and its transpose have the same cost as the normalized structure. The three and four-multiplier structures and their transposes are more expensive. The one-multiplier and its transpose have the fewest multiplies, although they require an extra add and a sign inversion compared to the other structures. 


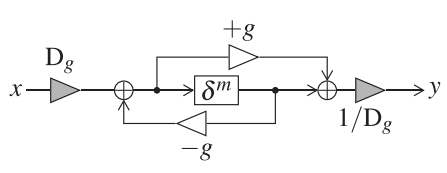

(a) Single Schroeder allpass filter.

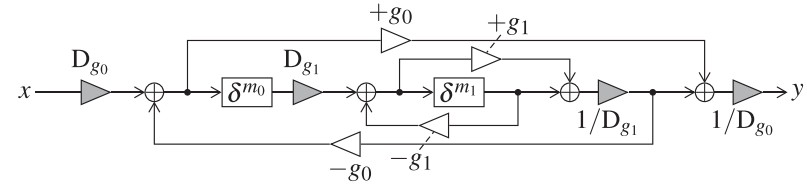

(b) Nested Schroeder allpass filter.

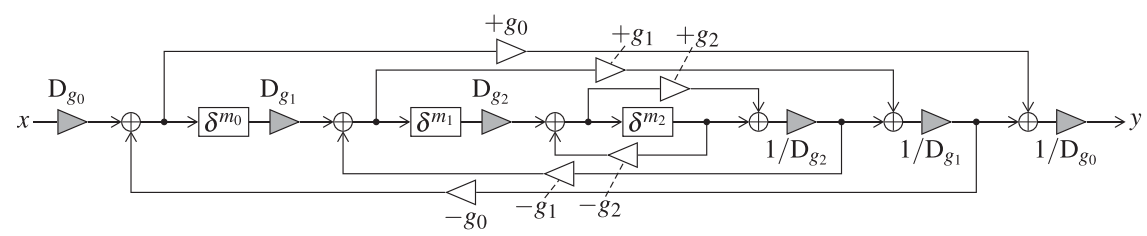

(c) Nested Schroeder allpass filter.

Fig. 7. Signal flow graphs for three energy-preserving Schroeder allpass filters.

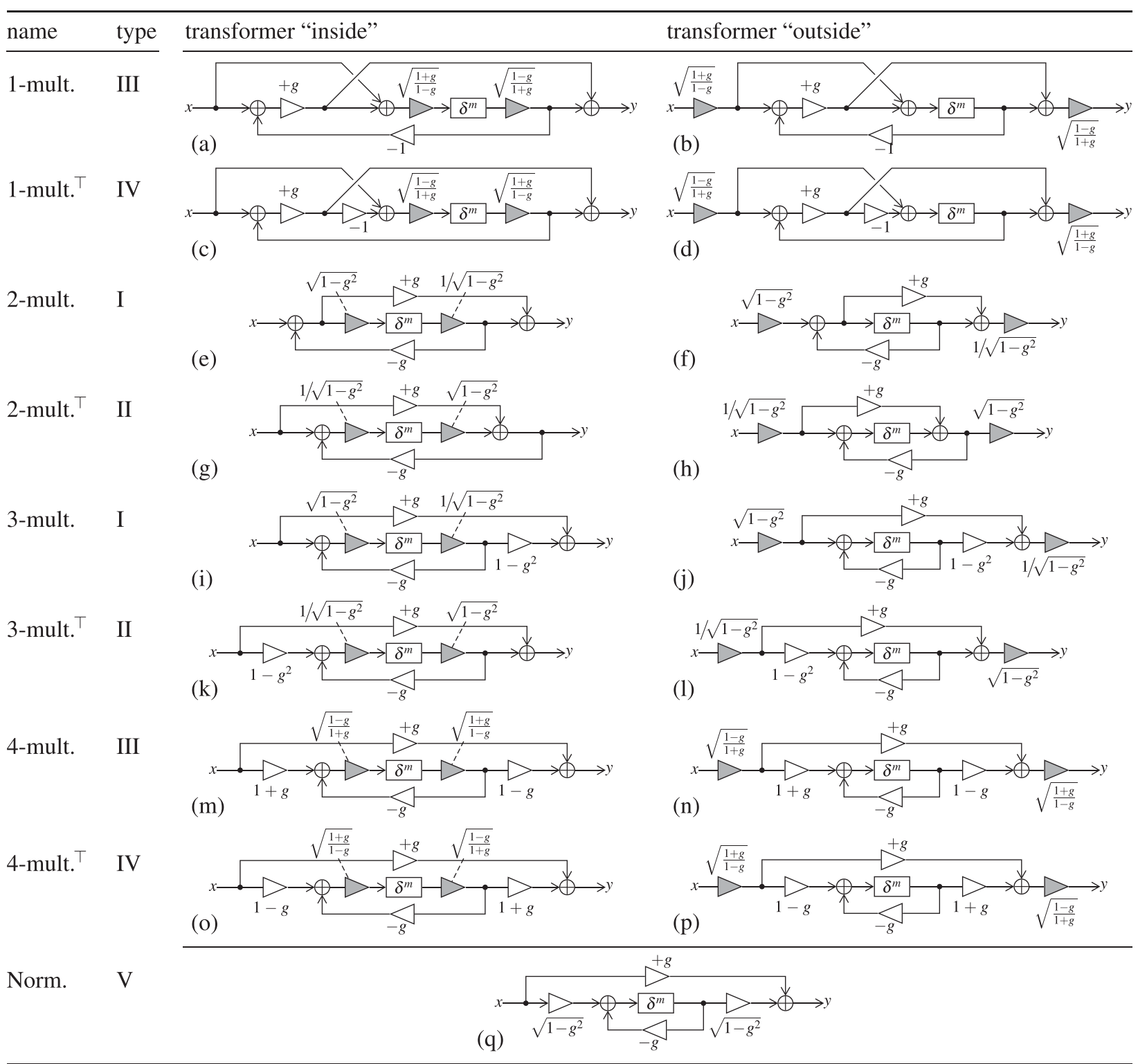

Fig. 8. Proposed energy-preserving time-varying (SISO) Schroeder allpass filter structures. 
Table 2. Implementation costs of proposed filter structures.

\begin{tabular}{lllllll}
\hline \hline Name & Type & $\times$ & + & Inv. & $\delta$ & Total op. \\
\hline 1-mult. / 1-mult. $^{\top}$ & III / IV & 3 & 3 & 1 & $M$ & $M+7$ \\
2-mult. / 2-mult. $^{\top}$ & I / II & 4 & 2 & 0 & $M$ & $M+6$ \\
3-mult. / 3-mult. $^{\top}$ & I / II & 5 & 2 & 0 & $M$ & $M+7$ \\
4-mult. / 4-mult. $^{\top}$ & III / IV & 6 & 2 & 0 & $M$ & $M+8$ \\
normalized & V & 4 & 2 & 0 & $M$ & $M+6$
\end{tabular}

It is likely that the most interesting structures are those with the lowest cost $\left(1-\right.$ mult. $\left.{ }^{(\top)}\right)$ and those resembling the common LTI embodiment (2-mult. $\left.{ }^{(\mathrm{T})}\right)$. We leave to future work any study of overflow, noise, and sensitivity of the different designs, in the spirit of Stilson's work on studying the different LTI allpass systems [54].

By ignoring the transformer multiplies we obtain a family of 9 different LTI allpass filter structures (not 17, since the "inside"/"outside" distinction does not apply in the LTI case). Several were already known in the literature. The original Schroeder allpass was a 3-mult. version (Fig. $8(\mathrm{i}) /(\mathrm{j})$, w/o transformers). Two-mult. versions were used, e.g., in the "Sampson box" at CCRMA [45, 55, 56], and these are the versions (Fig. 8(e)-8(h), w/o transformers) most commonly encountered in the literature today $[7,11$, 44]. Moorer also proposed a one-multiply variant [4] (Fig. 8(a)/(b), w/o transformers). Fig. 8(c)/(d), (k)/(l), and (m)/(n) appear to be novel.

Taking $M=1$, certain first-order versions of these structures are known in the literature. Fig. 8(a)-8(d) arises as a consequence of the "multiplier extraction" method [57]. Fig. $8(\mathrm{e}) /(\mathrm{f})$ is known $[10,58]$. Fig. $8(\mathrm{~g}) /(\mathrm{h})$ has been studied for its scaling properties [54]. Fig. 8(q) was proposed as an energy-preserving time-varying first-order allpass filter [17] and inspired the current work [31].

\section{MULTIPLIER SAVINGS}

We have derived energy-preserving versions of all known forms of the basic Schroeder allpass filter as well as novel ones, which can be nested and cascaded while still retaining their energy preservation property. In this section we point out some special cases that form filter structures with fewer multiplies: cascades with identical and inverse gains (SEC. 3.1) and up to four distinct gains (SEC. 3.2), a "leapfrog" multiplier-sharing arrangement (SEC. 3.3), nestings (SEC. 3.4), and a strategy based on periodic modulation (SEC. 3.5). None of these proposed strategies put any restrictions on delay line lengths. We assume $\rho=+1$ throughout $^{1} ; \rho=$ -1 appears not to be useful in any of these multiplier-saving cascades or nestings.

\subsection{Identical and Inverse-Multiplier Cascades}

For the structures with the transformer "outside," the multiplies $\xi$ (resp. $1 / \xi$ ) appear outside of any feedback loop, just after (resp. before) the input (resp. output). When two proposed filters with identical allpass gains $g$ are cascaded

\footnotetext{
${ }^{1} \rho$ is defined in the APPENDIX.
}

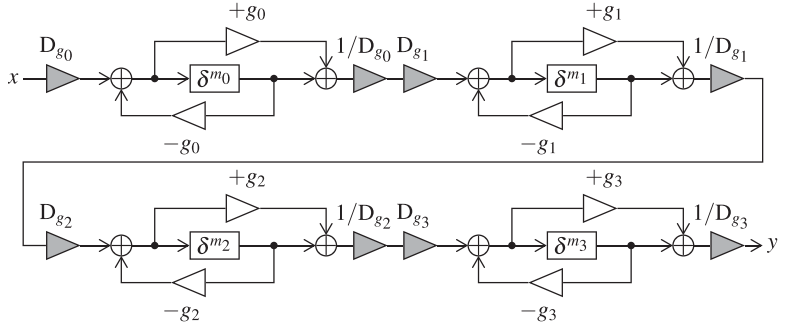

(a) Four cascaded Schroeder allpass filters.

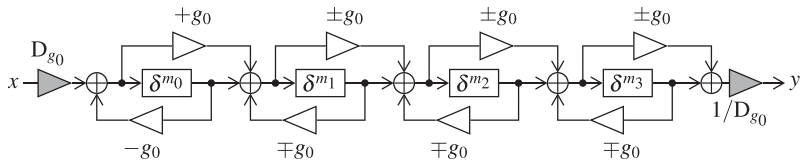

(b) Eliminating pairs of inverse multipliers, $g_{1}, g_{2}, g_{3}= \pm g_{0}$.

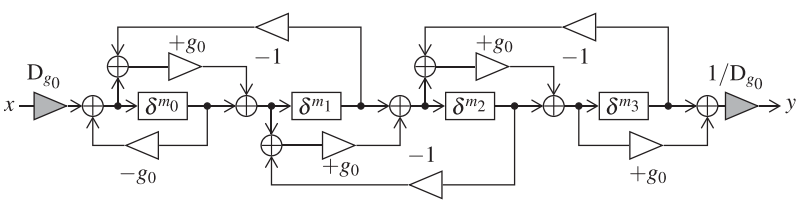

(c) Combining similar multipliers, $g_{1}, g_{2}, g_{3}=g_{0}$.

Fig. 9. An example of multiplier savings in cascades.

the reciprocal multiplies $\xi$ and $1 / \xi$ cancel out, saving two multiplies. This holds for Types I-IV. It does nothing for Type $\mathrm{V}$, where $\xi=1 / \xi=1$. An example is shown in Fig. 9(a) and 9(b), where all adjacent inverse multipliers in a cascade of four two-mult. (Type III) structures are eliminated. Although this is a special case it is a very common one. Most reverbs in the literature employing cascades of Schroeder allpasses use identical gains for each. ${ }^{2}$

For 2/3-multiply (Type I) cases and their transposes (Type II) this property also holds whenever $g_{i+1}=-g_{i}$. This occurs, e.g., in one of Schroeder's original reverbs [1], which has 5 allpasses in cascade with identical gains, up to a sign flip: $+0.7,-0.7,+0.7,+0.7,+0.7$.

In certain cases we can also eliminate pairs of multiplies by alternating $g$ 's sign as well as alternating between 1 mult. ${ }^{(T)}$ implementations (Type III/IV). If stage $i$ is Type III (resp. IV), switching to Type IV (resp. III) in stage $i+$ 1 allows the pair of multiplies to be eliminated when $g_{i+1}$ $=-g_{i}$. However no such property holds for the $2 / 3$-mult. (Type I) or its transpose (Type II).

Outside the reverb context a cascade of many identical first-order allpasses can be used in string modeling [18]. This previously cost 4 multiplies per sample of maximum delay: $4 N$ for $N$ stages. The proposed method reduces this down to $N+2$, using 1-mult. or 1-mult. ${ }^{\top}$, cutting ( $3 N-$ $2) / 4 N \approx 75 \%$ of the multiplies. The example in [18] has $N=126$, so here the proposed method would yield significant savings (376 multiplies per time step).

\footnotetext{
${ }^{2} \mathrm{~A}$ summary of the number of allpasses with identical gains in cascade for various reverbs is shown in Table 4.
} 


\subsection{Generalized Multiplier-Saving Cascades}

Generalizing the property from SEC. 3.1 allows us to perform the same multiplier-saving operation of canceling adjacent multiplies while not necessarily resorting solely to identical or inverse gains. Recalling Table 1, notice that $\xi_{i+1}$ $=\xi_{i}$ can be satisfied in certain cases by choosing different types and values for $g$ for each stage, leading to canceled multipliers.

Table 3 shows a set of functions, $f_{\text {type }_{i}, \text { type }_{i+1}}()$, which choose a multiplier-saving gain $g_{i+1}$ for stage $i+1$ based on the gain $g_{i}$ of stage $i . f_{\mathrm{III}, \mathrm{I}}^{ \pm}, f_{\mathrm{III}, \mathrm{II}}^{ \pm}, f_{\mathrm{IV}, \mathrm{I}}^{ \pm}$, and $f_{\mathrm{III}, \mathrm{II}}^{ \pm}$are multifunctions with positive and negative branches.

Several cases are degenerate in one way or another. First there is no combination of $g_{i}$ and $g_{i+1}$ (other than $g_{i}=g_{i+1}$ $=0$ ) that allows transitioning to or from the normalized structure (Type V). Second $f_{\mathrm{I}, \mathrm{II}}$, and $f_{\mathrm{III}, \mathrm{I}}$ have domains and ranges of $\{0\}$ - they are only valid for the useless case of $g_{i}=0$ (a trivial cascade of two pure delay lines, non-timevarying). Also, not all gains $g_{i}$ map to a multiplier-canceling $g_{i+1}$, restraining the valid domain of the different $f()$.

For example, starting with $g_{0}=0.7$ can yield a cascade:

Type I: $\quad g_{0}[n]=+0.7$,

Type III: $\quad g_{1}[\mathrm{n}] \approx+0.3245=f_{\mathrm{I}, \mathrm{III}}\left(g_{0}[n]\right)$,

Type IV: $\quad g_{2}[n] \approx-0.3245=f_{\text {III,IV }}\left(g_{1}[n]\right)$,

Type I: $\quad g_{3}[n]=-0.7=f_{\text {IV }}^{-}\left(g_{2}[n]\right)$.

As it happens in this example we note that any cascade will necessarily obey the following two constraints. First, due to the need for compatible domains and ranges between stages, Type I and II structures cannot both appear in the same multiplier-saving cascade, even if separated by other types. Second, the technique described here never leads to more than four distinct gain values.

\subsection{Leapfrog Cascade}

In cases with adjacent cascaded allpass stages with identical gains there are adjacent branch points or sums. In these cases, pairs of multiplies from adjacent stages can be combined to reduce the total multiplier count, down to as low as $N+3$ for a cascade of $N$ stages.

An example of this is shown in Fig. 9(b) and 9(c). Note that in Fig. 9(c) every other stage has been flipped vertically to keep the signal flow graph planar and easy to read. This structure resembles the classic "Leapfrog" filter structure from active filter synthesis [59]. Interestingly, besides the presence of the two multiplies, at the input and output of the whole structure, which accomplish the energy normalization, this and the same idea applied to 2 -mult. $^{\top}$ yield identical structures to the "delay-sharing" allpass cascades proposed by Mitra and Hirano [57].

\subsection{Nesting}

In nesting structures $[11-14,16]$ we can also eliminate transformer multipliers. To expose multipliers to their inverses we alternate between "inside" and "outside" each nesting. In a nested structure the delay line is replaced with the cascade of a Schroeder allpass filter and at least a onesample delay, the delay being necessary for realizability, to avoid a "delay-free loop." The delay makes it so that 1

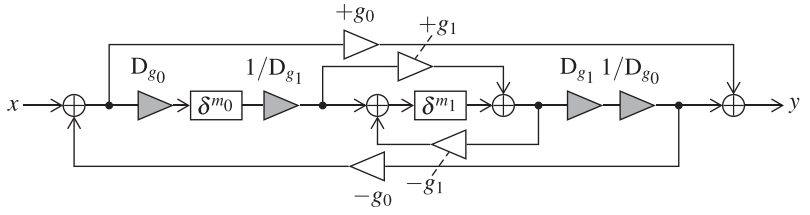

(a) Two nested Schroeder allpass filters.

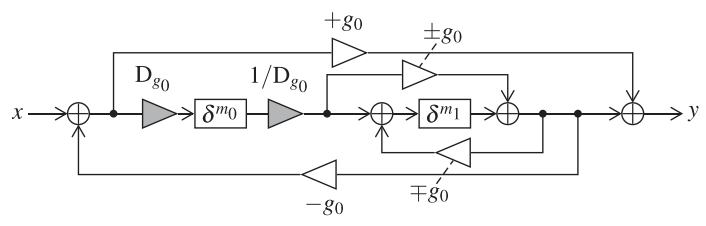

(b) Eliminating pairs of inverse multipliers, $g_{1}= \pm g_{0}$.

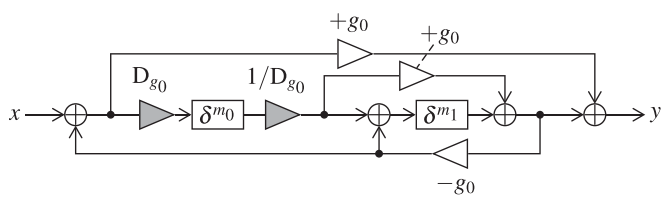

(c) Combining similar multipliers, $g_{1}=g_{0}$.

Fig. 10. An example of multiplier savings in nestings.

pair of reciprocal multipliers are not actually adjacent, only giving us the chance to save 1 multiplier per nesting, not 2. An example of nesting a 2-mult. ${ }^{\top}$ (Type II) inside of a 2-mult. (Type I) structure is shown in Fig. 10(a).

Valid gains in nestings are handled very similarly to cascades. However, to save a multiplier in a nesting, we need to solve $\xi_{i+1}=1 / \xi_{i}$ rather than $\xi_{i+1}=\xi_{i}$. The solutions can be described by Table 3 after simply swapping the columns for both Types I and II, and III and IV.

Furthermore, similar to the "leapfrog" arrangement of cascades previously discussed, in cases where adjacent nested stages with identical or inverse gains also lead to adjacent branch points or sums, pairs of multiplies from adjacent stages can be combined to lower the multiplication count. An example of this is shown for the same nesting example, in the case that $g_{1}=g_{0}$, in Fig. 10(b) and 10(c).

\subsection{Periodic Gain Modulation}

An additional restrictive way to save multiplies is to modulate gains by specific periodic functions. When $g[n]$ is periodic in $M$ the two transformer multiplies cancel out, saving two multiplies. This can be seen by considering the "inside" proposed structures (Fig. 8). An equivalent filter is obtained by "pushing" $\xi[n]$ through the length- $M$ delay line, giving a composite multiplier $\xi[n-M] / \xi[n]$, which cancels out when $g[n]=g[n-M], \forall n$.

Alternatively, consider (19), the energy-preserving timevarying Schroeder allpass filter's difference equation. When $g[n]=g[n-M] \forall n$, we get $\mathrm{D}_{g[n]} / \mathrm{D}_{g[n-M]}=1$, and the difference equation reduces to (34), the difference equation for a linear time-invariant Schroeder allpass filter. 
Table 3. Gain $g_{i+1}$ of the $(i+1)$ th stage that eliminates reciprocal multiplies in a cascade, in terms of the previous $\left(i\right.$ th) stage's gain $g_{i}$. (Multi)function domains and ranges are shown as $\{$ domain $\} \longrightarrow\{$ range $\}$ if different from $]-1,+1[$. For the case of nested structures, swap columns "Type I" and "Type II," and swap columns "Type III" and "Type IV."

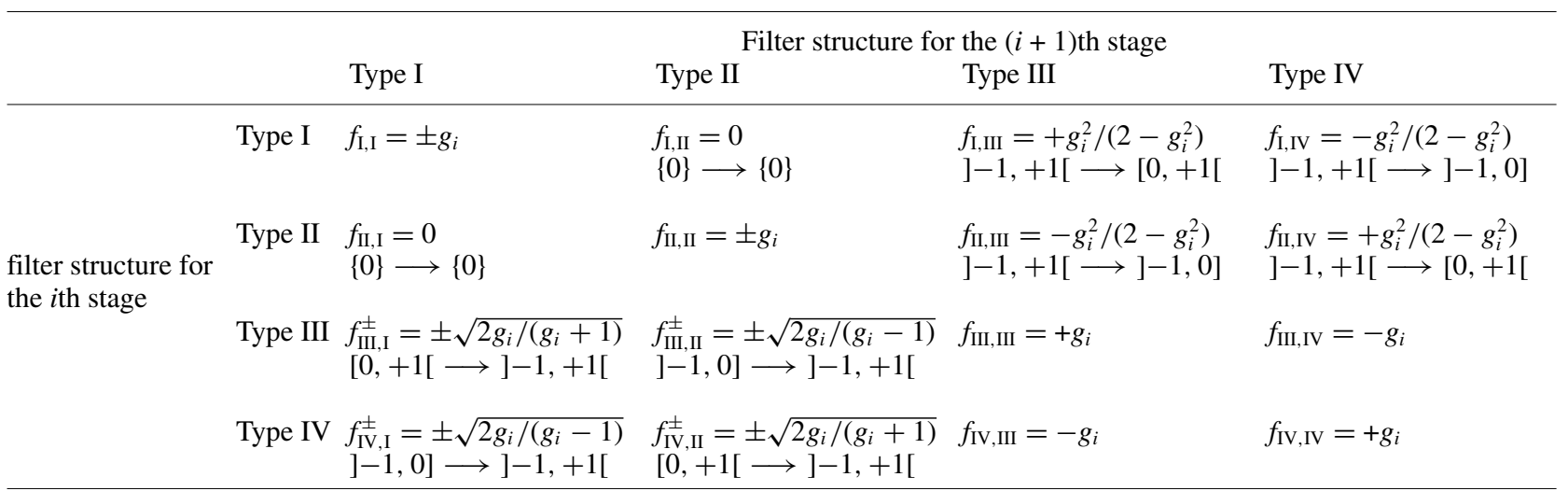

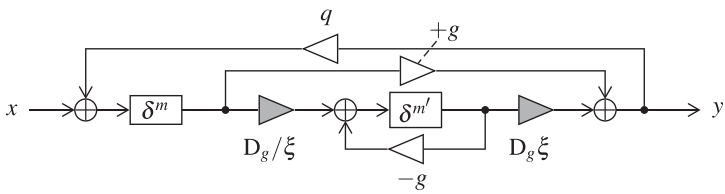

(a) Allpass 1-FDN.

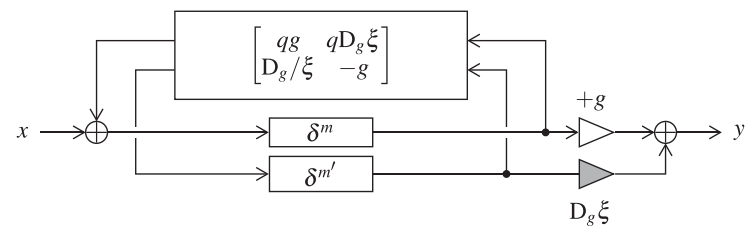

(b) Redrawn as standard 2-FDN.

Fig. 11. Allpass 1-FDN to standard 2-FDN conversion.

\section{SISO STABILITY CASE STUDY}

In this section a case study demonstrates how the proposed structures can stabilize an allpass 1-FDN prototype. Experimental results in [31] show that this structure preserves energy down to numerical precision. Schlecht introduced the idea of rewriting different digital reverb structures as FDNs $[8,60]$ so that, e.g., they can be studied using Laroche's time-varying stability criteria [61] or concepts like unilosslessness [53].

Consider a SISO "allpass 1-FDN" in the sense of a standard 1-FDN augmented with an allpass filter in cascade with its delay line, with allpass gain $g$ and FDN feedback gain $q$, as shown in Fig. 11(a). Here the Schroeder allpass filter is realized by the generalized block (Fig. 4(a)) without any corresponding transformer. Here the case $\xi=1$ represents any realization of the proposed structures and the cases $\xi \neq 1$ represent others. For instance $\xi=1 / \mathrm{D}_{g}$ would represent a realization with a classic Type I structure, i.e., one un-treated by our proposed techniques.

Assume that we are designing an FDN "reference filter" $[3,49]$ or "prototype" [50] - an FDN that is supposed to be marginally stable, ringing forever with a constant energy in response to an impulse input. To make a reference filter whose energy cannot vary over time as $g$ is changed, the feedback matrix should be unitary. Considering that the feedback matrix in the allpass $1-\mathrm{FDN}$ is simply $[q]$, this means that $q= \pm 1$.

Rewriting the allpass 1-FDN as a standard 2-FDN in delay state-space form, as shown in Fig. 11(b), yields

$$
\begin{aligned}
\mathbf{A} & =\left[\begin{array}{cc}
q g & q \mathrm{D}_{g} \xi \\
\mathrm{D}_{g} / \xi & -g
\end{array}\right], & \left.\mathbf{B}=\left[\begin{array}{l}
1 \\
0
\end{array}\right], \quad \mathbf{w}=\Delta{ }^{[m} m^{\prime}\right]^{\top} \mathbf{u}, \\
\mathbf{C}=\left[\begin{array}{lll}
g & \xi \mathrm{D}_{g}
\end{array}\right], & \mathbf{D}=[0] . &
\end{aligned}
$$

Again, we would like the feedback matrix $\boldsymbol{A}$ to be unitary. Keeping in mind the already established constraints $|g|<1$ and $q= \pm 1$, we find that a sufficient criteria for unitarity of $\boldsymbol{A}$ is $\xi=1$. This means that any of the proposed structures in SEC. 2 (Fig. 8) ensure that varying $g$ over time does not affect the stored energy in the system. Considering the weaker constraint of unilosslessness, $\boldsymbol{A}$ is unilossless for any $\xi$. Again keeping in mind the already established constraints on $g$ and $q, \boldsymbol{A}$ is diagonally similar to $\left[\begin{array}{cc} \pm 1 & 0 \\ 0 & 1\end{array}\right] \boldsymbol{U}(g)$ by $\boldsymbol{P}=\left[\begin{array}{cc}1 & 0 \\ 0 & 1 / \xi\end{array}\right]$. This was shown for the particular case of a 2-mult. ${ }^{\top}$ (type II, $\xi=1 / \mathrm{D}_{g}$ ) Schroeder allpass filter in [53]. In sum, $\boldsymbol{A}$ is unilossless but only unitary for $\xi=1$.

We can also look at the stability of this system from the standpoint of [61]. We have shown that the the reference filter is marginally stable when $q= \pm 1$; now we contract that to $|q|<1$. Intuitively this should create a bounded-input bounded-output (BIBO) stable system, just as in the timeinvariant case. We study two cases: the somewhat restrictive case of all delay line lengths equal to one, for which we prove BIBO stability using Laroche's criterion, and the more general case of delay lines of any length, for which we have strong evidence of stability but no formal proof.

If we set the delay line lengths $m, m^{\prime}=1$, we can apply Laroche's proof directly. Laroche showed that if the norm (largest singular value) of the state transition matrix in a standard state space system is less than one at every time step, i.e., $\|\boldsymbol{A}[n]\|<1 \forall n$, then the structure is BIBO stable. Setting our two delay line lengths to 1 means that $\boldsymbol{A}$ becomes identical to the standard state transition matrix. Unfortunately, as Laroche found in studying a normalized ladder filter, we find that $\boldsymbol{A}$ has singular values of 1 and $q$. 
So $\|\boldsymbol{A}\|=1$, and we cannot apply this theorem directly to prove BIBO stability.

However we can instead study a generalization, the norm of the transition matrix corresponding to two time steps, and show that the norm of this matrix is less than one, i.e., $\|\boldsymbol{A}[n+1] \boldsymbol{A}[n]\|<1$. Setting $\xi=1$ (e.g., using the proposed Schroeder allpass structure rather than the classic one), this can be shown using a similar procedure to the one Laroche uses in the appendix of [61]. Unlike Laroche, we have not assumed that $g[n]=g[n+1]$, nor that $\boldsymbol{A}[n]=\boldsymbol{A}[n+1]$. So we achieve the proof of BIBO stability without any constraint that $g$ does not vary over the two time steps. In this sense we have also expanded slightly on Laroche's proof (which assumes $\boldsymbol{A}[n]=\boldsymbol{A}[n+1]$ ).

More generally, allowing $m \geq 1$ and $m^{\prime} \geq 1$, we arrive at a much more complicated state transition matrix $\boldsymbol{A} \in \mathbb{R}^{\left(m+m^{\prime}\right) \times\left(m+m^{\prime}\right)}$ for which it is not simple to study $\|\boldsymbol{A}[n]\|$ or $\|\boldsymbol{A}[n+1] \boldsymbol{A}[n]\|$ in closed form. Furthermore it appears that when $2<m+m^{\prime}$, even $\|\boldsymbol{A}[n+1] \boldsymbol{A}[n]\|=1$, so two time steps are not sufficient to prove BIBO stability. From experimenting with a number of delay line lengths we conjecture that the number of time steps we must consider to show BIBO stability is $H=m+m^{\prime}$, i.e., we would need to prove that $\left\|\prod_{\eta=0}^{\eta=H} \boldsymbol{A}[n+\eta]\right\|<1$. Our tests have shown us that setting $\xi=1$ is sufficient to achieve this, while $\xi \neq 1$ is not. This shows that the proposed structures create BIBO-stable allpass FDNs while classical Schroeder allpass filters do not. Unfortunately we do not at this time have a formal proof.

Along these lines we note that Schlecht [8] has shown that applying a constant gain $|\nu|<1$ to a feedback matrix that is unitary at every time step is sufficient to stabilize the structure. This is related to but not precisely the same as setting $|q|<1$. Instead it forms a feedback matrix $\boldsymbol{A}=\left[\begin{array}{cc}-v g & v \mathrm{D}_{g} \\ v \mathrm{D}_{g} & -v g\end{array}\right]$, which disrupts the allpass nature of the Schroeder filter by adding frequency-independent "absorption" [7, 49], giving it a transfer function of (assuming LTI for a moment for notational simplicity)

$$
H(z)=\frac{g+\nu z^{-m}}{1+g \nu z^{-m}} .
$$

\section{PROPOSED MIMO STRUCTURES}

So far we have focused on creating energy-preserving time-varying SISO systems, particularly Schroeder allpass filters and generalizations (nestings and cascades). Now we show how the same techniques can be used to create energy-preserving time-varying MIMO systems, particularly generalizations of a Gerzon reverberator. Because of the added complexity of these systems we leave the particular ways that unitary matrices $\boldsymbol{U}(\boldsymbol{G})$ are realized and strategies for multiplier savings for future work but rather just focus on the structure of several particular versions of $\boldsymbol{G}$.

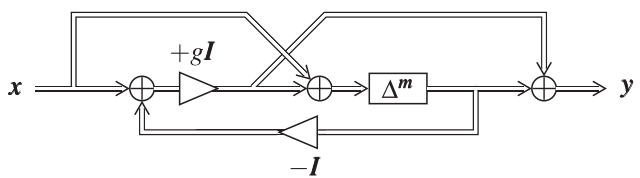

Fig. 12. Poletti's MIMO allpass reverberator.

\subsection{MIMO Building Blocks}

The building blocks of MIMO ( $N$-input, $N$-output) allpass structures are related to those for SISO structures. Rather than individual delay lines $\delta^{m}$ we have parallel arrangements of delay lines $\Delta^{m}, \boldsymbol{m} \in \mathbb{R}^{N \times 1}$.

Rather than using only $2 \times 2$ unitary matrices as we did for the Schroeder filters we use larger unitary matrices. In analogy to the SISO case where an allpass gain with $|g|<1$ leads to a $2 \times 2$ unitary matrix $\boldsymbol{U}(g)$ as in (7), in the MIMO case we have a contraction $\boldsymbol{G} \in \mathbb{R}^{N \times N}$, forming a unitary dilation $\boldsymbol{U}(\boldsymbol{G}) \in \mathbb{R}^{2 N \times 2 N}$, as in (5).

We could also use some of the many other unitary matrix parameterizations that exist and are well known in the reverb literature [51]. However we do always need to be mindful of stability, ensuring that the norm of the upper-left portion of the unitary matrix corresponding to the feedback is less than one at each time step for stability.

\subsection{MIMO Recipe}

We can again use the rules from SEC. 1.5 to combine these building blocks into new energy-preserving systems. But first we review two reverbs from the literature. Consider the reverbs [9, 62] of Gerzon ("G," Fig. 1(b)) and Poletti ("P," Fig. 12), which have system matrices

$$
\boldsymbol{S}_{\mathrm{G}}=\left[\begin{array}{cc}
-g \boldsymbol{I} & \boldsymbol{I} \\
\left(1-g^{2}\right) \boldsymbol{I} & g \boldsymbol{I}
\end{array}\right], \quad \boldsymbol{S}_{\mathrm{P}}=\left[\begin{array}{cc}
-g \boldsymbol{I} & (1+g) \boldsymbol{I} \\
(1-g) \boldsymbol{I} & g \boldsymbol{I}
\end{array}\right] .
$$

These are both particular cases of $\boldsymbol{L}(g, \xi) \otimes \boldsymbol{I}$, where $\otimes$ is the Hadamard product. Their multiplier structure is simply a vector version of two versions of an LTI Schroeder allpass filter: the 3-mult. and 1-mult., respectively.

$\boldsymbol{S}_{\mathrm{G}}$ and $\boldsymbol{S}_{\mathrm{P}}$ are both diagonally similar to $\boldsymbol{U}(g \boldsymbol{I})$ by $\left[\begin{array}{cc}1 & 0 \\ 0 & \mathrm{D}_{g}\end{array}\right] \otimes \boldsymbol{I}$ resp. $\left[\begin{array}{cc}1 & 0 \\ 0 & \mathrm{D}_{g} /(1+g)\end{array}\right] \otimes \boldsymbol{I}$. This means that each matrix is unilossless [53]. However neither one is unitary.

It can be shown that any system matrix

$$
\boldsymbol{S}=\left[\begin{array}{cc}
-g & \mathrm{D}_{g} \xi \\
\mathrm{D}_{g} / \xi & g
\end{array}\right] \otimes \boldsymbol{I}
$$

is diagonally similar to $\boldsymbol{U}(g \boldsymbol{I})$ by $\left[\begin{array}{ll}1 & 0 \\ 0 & \xi\end{array}\right] \otimes \boldsymbol{I}$. So all system matrices in the form of $S$ are unilossless but not unitary unless $\xi=1$. Although these structures (with $\xi \neq 1$ ) would not enjoy good energy-preservation properties under timevarying $g$, we mention them because they should work fine for LTI applications and appear not to have been widely reported, except for Gerzon's original and Poletti's.

This allows all of the structures presented in the earlier SISO allpass section to be readily converted to MIMO form just by replacing each scalar gain by a diagonal matrix of 


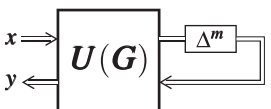

(a) Recipe

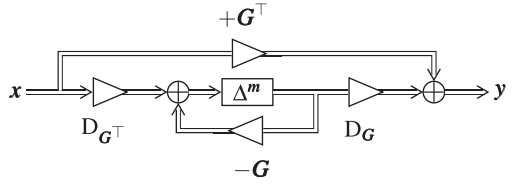

(b) Signal flow graph.
Fig. 13. Forming an energy-preserving version of Gerzon's reverberator from the building blocks.

identical scalar gains, similar to Gerzon's original concept [9], but with better time-varying properties.

Similar results hold if the diagonal matrix of identical gains $g \boldsymbol{I}$ is replaced by a diagonal matrix of distinct gains $\underline{\boldsymbol{G}}=\operatorname{diag}\left(g_{0}, g_{1}, \ldots, g_{N-1}\right)$, and the diagonal matrices of identical gains $\xi \boldsymbol{I}$ and $1 / \xi \boldsymbol{I}$ are replaced by diagonal matrices of distinct gains $\underline{\underline{\Xi}}=\operatorname{diag}\left(\xi_{0}, \xi_{1}, \ldots, \xi_{N-1}\right)$ and $\underline{\boldsymbol{\Xi}}^{-1}$. In this case any system matrix

$$
S_{\mathrm{d}}=\left[\begin{array}{cc}
-\underline{G} & \mathrm{D}_{\underline{G}} \underline{\Xi} \\
\underline{\Xi}^{-1} \mathrm{D}_{\underline{G}} & \underline{\underline{G}}
\end{array}\right]
$$

is diagonally similar to $\boldsymbol{U}(\underline{\boldsymbol{G}})$ by $\underline{\boldsymbol{P}}=\left[\begin{array}{cc}\boldsymbol{I} & \mathbf{0} \\ \mathbf{0} & \boldsymbol{\Xi}^{-1}\end{array}\right]$. So all system matrices in the form of $\boldsymbol{S}_{\mathrm{d}}$ are unilossless but not unitary unless $\left|\xi_{n}\right|=1, \forall n \in\{0,1, \ldots, N-1\}$. This generalizes Gerzon's approach to a wider class of structures and also imbues them with the energy-preservation property under time-varying conditions.

To produce a MIMO energy-preserving system we terminate a block $\boldsymbol{U}(\boldsymbol{G}) \in \mathbb{R}^{2 M \times 2 M}$, parameterized by $\boldsymbol{G} \in$ $\mathbb{R}^{M \times M}$, on a parallel arrangement of delay lines $\Delta^{m}$, $\boldsymbol{m} \in \mathbb{R}^{M \times 1}$. This is defined by $\mathrm{F}\left(\boldsymbol{U}(\boldsymbol{G}), \Delta^{\boldsymbol{m}}\right)$, as shown in Fig. 13(a), and leads to the signal flow graph shown in Fig. 13(b). In the same way that Gerzon's reverb generalized Schroeder allpasses, this structure generalizes the proposed energy-preserving Schroeder allpass structures.

This generalizes Gerzon's design, replacing the single gain $g$ with a full matrix of gains $\boldsymbol{G}$, with $\boldsymbol{G}$ contractive $(\|\boldsymbol{G}\|<1)$. This gives us a system matrix

$$
\boldsymbol{S}=\boldsymbol{U}(\boldsymbol{G})=\left[\begin{array}{cc}
-\boldsymbol{G} & \mathrm{D}_{\boldsymbol{G}^{\top}} \\
\mathrm{D}_{\boldsymbol{G}} & \boldsymbol{G}^{\top}
\end{array}\right] .
$$

The big difference w.r.t. (23)-(24) is that we see $\boldsymbol{G}^{\top}$, not $\boldsymbol{G}$, in the right partition. This $\boldsymbol{S}$ is unitary by construction; hence the reverberator constructed from it will enjoy the energy-preservation property. The main reason to care about more complicated structures like this is that the echo density will build up faster with a dense feedback matrix than the diagonal feedback matrices of (23)-(24).

As in the SISO case these can be combined into more complicated structures, e.g., nestings and cascades $[3,9$, 49]. Replacing the parallel arrangement of delay lines $\Delta^{m}$ with any $M \times M$ paraunitary system is common [5]. It is probably more common than nestings in the Schroeder allpass filters, since with the classic Gerzon design, parameterized by $g \boldsymbol{I}$, it is essentially a parallel arrangement of Schroeder allpass filters, unless the delay lines $\Delta^{m}$ are replaced by a more complicated energy-preserving system (classically, paraunitary) to mingle the signals among channels.

These structures are related to a type of FDN recently proposed by Schlecht. In [34] he showed how to create $M$ input, $M$-output FDNs with $N$ delay lines that are allpass by construction. He treats the problem of "orthogonal completion," or how create a matrix $\boldsymbol{U} \in \mathbb{R}^{(M+N) \times(M+N)}$ from its $N$ $\times N$ upper left partition (subject to some admissibility constraints on that partition), yielding a structure $\mathrm{F}\left(\boldsymbol{U}, \Delta^{m}\right)$. Based on our development here, structures using his "orthogonal completion" will be energy-preserving under time varying conditions as well.

As an alternate to showing that the resulting structures are allpass due to the rules for combining energy-preserving systems, we can show that this property holds through algebra. We can begin with the transfer function

$$
\boldsymbol{H}(z)=\boldsymbol{G}+\mathrm{D}_{\boldsymbol{G}^{\mathrm{H}}} \boldsymbol{Z}(z)\left(\boldsymbol{I}+\boldsymbol{G}^{\mathrm{H}} \boldsymbol{Z}(z)\right)^{-1} \mathrm{D}_{\boldsymbol{G}},
$$

where $\boldsymbol{G}$ is a contraction $(\|\boldsymbol{G}\| \leq 1)$ and $\boldsymbol{Z}(z)$ is a paraunitary matrix $(\tilde{\boldsymbol{Z}}(z) \boldsymbol{Z}(z)=\boldsymbol{I})$ with every entry causal (either zero or with at least one delay $z^{-1}$ ). To show that this is an allpass transfer function we must prove that $\tilde{\boldsymbol{H}}(z) \boldsymbol{H}(z)=\boldsymbol{I}$. Suppressing $(z)$ for compactness, we have

$$
\tilde{\boldsymbol{H}} \boldsymbol{H}=\boldsymbol{G}^{\mathrm{H}} \boldsymbol{G}+\boldsymbol{G}^{\mathrm{H}} \boldsymbol{H}_{2}+\tilde{\boldsymbol{H}}_{2} \boldsymbol{H}_{2}+\tilde{\boldsymbol{H}}_{2} \boldsymbol{G}
$$

where

$$
\begin{aligned}
\boldsymbol{H}_{2} & =\mathrm{D}_{\boldsymbol{G}^{\mathrm{H}}} \boldsymbol{Z}\left(\boldsymbol{I}+\boldsymbol{G}^{\mathrm{H}} \boldsymbol{Z}\right)^{-1} \mathrm{D}_{\boldsymbol{G}}, \\
\tilde{\boldsymbol{H}}_{2} & =\mathrm{D}_{\boldsymbol{G}}(\boldsymbol{I}+\tilde{\boldsymbol{Z}} \boldsymbol{G})^{-1} \tilde{\boldsymbol{Z}} \mathrm{D}_{\boldsymbol{G}^{\mathrm{H}}} .
\end{aligned}
$$

Using the fact that $\mathrm{D}_{\boldsymbol{G}^{\mathrm{H}}} \boldsymbol{G}=\boldsymbol{G} \mathrm{D}_{\boldsymbol{G}}$ and $\mathrm{D}_{\boldsymbol{G}} \boldsymbol{G}^{\mathrm{H}}=\boldsymbol{G}^{\mathrm{H}} \mathrm{D}_{\boldsymbol{G}^{\mathrm{H}}}$ [63] to rearrange $\tilde{\boldsymbol{H}}_{2} \boldsymbol{G}$ and $\boldsymbol{G}^{\mathrm{H}} \boldsymbol{H}_{2}$, all terms on the righthand side of (27) are now of the form $\mathrm{D}_{G} \ldots \mathrm{D}_{G}$. Then subtracting $\boldsymbol{G}^{\mathrm{H}} \boldsymbol{G}$ from both sides of the unitarity condition $\boldsymbol{I}=\tilde{\boldsymbol{H}}(z) \boldsymbol{H}(z)$, we come to

$$
\begin{aligned}
& \boldsymbol{I}-\boldsymbol{G}^{\mathrm{H}} \boldsymbol{G}=\boldsymbol{G}^{\mathrm{H}} \boldsymbol{H}_{2}+\tilde{\boldsymbol{H}}_{2} \boldsymbol{H}_{2}+\tilde{\boldsymbol{H}}_{2} \boldsymbol{G} \\
& =\mathrm{D}_{\boldsymbol{G}}\left[(\boldsymbol{I}+\tilde{\boldsymbol{Z}} \boldsymbol{G})^{-1} \tilde{\boldsymbol{Z}} \boldsymbol{G}\right. \\
& +(\boldsymbol{I}+\tilde{\boldsymbol{Z}} \boldsymbol{G})^{-1} \tilde{\boldsymbol{Z}} \mathrm{D}_{\boldsymbol{G}^{\mathrm{H}}}^{2} \boldsymbol{Z}\left(\boldsymbol{I}+\boldsymbol{G}^{\mathrm{H}} \boldsymbol{Z}\right)^{-1} \\
& \left.+\boldsymbol{G}^{\mathrm{H}} \boldsymbol{Z}\left(\boldsymbol{I}+\boldsymbol{G}^{\mathrm{H}} \boldsymbol{Z}\right)^{-1}\right] \mathrm{D}_{\boldsymbol{G}} .
\end{aligned}
$$

Now, since the left-hand side of the above is simply $\mathrm{D}_{G}^{2}$, if we left-multiply everything by $(\boldsymbol{I}+\tilde{\boldsymbol{Z}} \boldsymbol{G}) \mathrm{D}_{\boldsymbol{G}}^{-1}$ and rightmultiply everything by $\mathrm{D}_{\boldsymbol{G}}^{-1}\left(\boldsymbol{I}+\boldsymbol{G}^{\mathrm{H}} \boldsymbol{Z}\right)$, we have

$$
\begin{aligned}
& \boldsymbol{I}+\tilde{\boldsymbol{Z}} \boldsymbol{G}+\boldsymbol{G}^{\mathrm{H}} \boldsymbol{Z}+\tilde{\boldsymbol{Z}} \boldsymbol{G} \boldsymbol{G}^{\mathrm{H}} \boldsymbol{Z} \\
& \quad=\tilde{\boldsymbol{Z}} \boldsymbol{G}+\tilde{\boldsymbol{Z}} \boldsymbol{G} \boldsymbol{G}^{\mathrm{H}} \boldsymbol{Z}+\tilde{\boldsymbol{Z}} \mathrm{D}_{\boldsymbol{G}^{\mathrm{H}}}^{2} \boldsymbol{Z}+\boldsymbol{G}^{\mathrm{H}} \boldsymbol{Z}+\tilde{\boldsymbol{Z}} \boldsymbol{G} \boldsymbol{G}^{\mathrm{H}} \boldsymbol{Z} .
\end{aligned}
$$

Dropping the common terms, left-multiplying by $\boldsymbol{Z}$, right-multiplying by $\tilde{Z}$, and substituting $\mathrm{D}_{\boldsymbol{G}^{\mathrm{H}}}$ by its definition yields

$$
\boldsymbol{I}=\boldsymbol{I}-\boldsymbol{G} \boldsymbol{G}^{\mathrm{H}}+\boldsymbol{G} \boldsymbol{G}^{\mathrm{H}}
$$

\section{MIMO STABILITY CASE STUDY}

In this section we present a few case studies demonstrating how the proposed techniques can stabilize reverberator structures under time-varying conditions. Building on the 


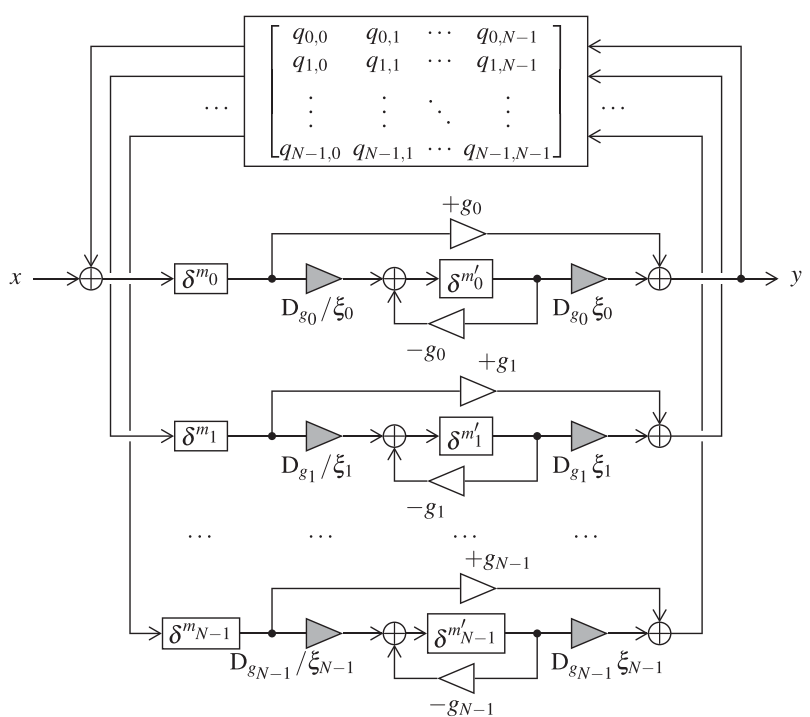

(a) Allpass $N$-FDN.

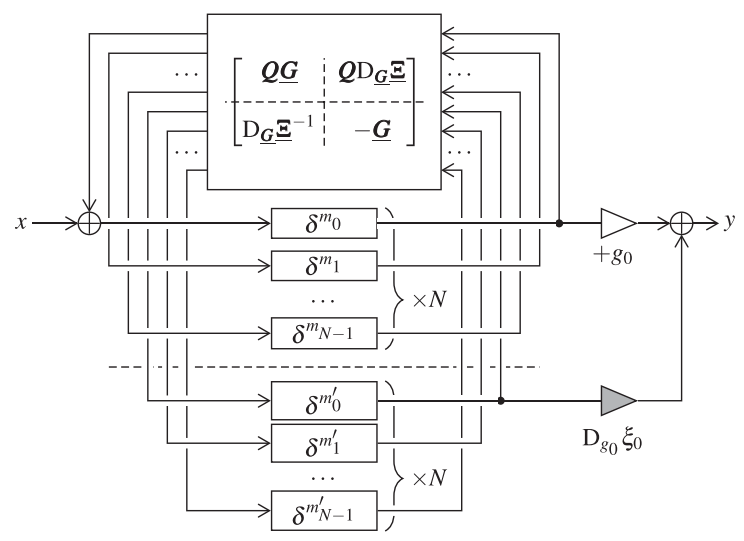

(b) Redrawn as standard $2 N-F D N$.

Fig. 14. Allpass $N$-FDN to standard $2 N$-FDN conversion.

results of SEC. 4, we show how a particular allpass 2-FDN prototype, which was previously shown to be unstable under time-varying coefficients, is stabilized. We also show an allpass $N$-FDN prototype, for general $N$, is stabilized using the proposed technique.

\subsection{Correcting a Particular 2-FDN}

In [8] Schlecht shows a particular allpass 2-FDN with time-varying gain $g$, which is not BIBO stable. In this section we show how the proposed techniques correct it, ensuring that it is BIBO stable.

Consider the allpass FDN shown in Fig. 14, with $N=2$, with the unitary feedback matrix $\boldsymbol{Q}=\left[\begin{array}{ll}0 & 1 \\ 1 & 0\end{array}\right]$, all delay line lengths $m_{0}, m_{1}, m_{0}^{\prime}, m_{1}^{\prime}=1$, and allpass gains

$$
g_{0}[n]=0.5, \quad g_{1}[n]=\left\{\begin{array}{ll}
-0.9, & n \text { odd } \\
0, & n \text { even }
\end{array} .\right.
$$

The state transition matrix is thus

$$
\boldsymbol{A}=\left[\begin{array}{cccc}
0 & g_{1} & 0 & \mathrm{D}_{g_{1}} \xi_{1} \\
g_{0} & 0 & \mathrm{D}_{g_{0}} \xi_{0} & 0 \\
\mathrm{D}_{g_{0}} / \xi_{0} & 0 & -g_{0} & 0 \\
0 & \mathrm{D}_{g_{1}} / \xi & 0 & -g_{1}
\end{array}\right] .
$$

For a 2-mult. ${ }^{\top}$ Schroeder [8], $\|\boldsymbol{A}[n]\|>1, \forall n$ and $\|\boldsymbol{A}[n] \boldsymbol{A}[n+1]\|>1, \forall n$. So the structure is not BIBO stable, nor is it marginally stable- the property that it should have as an FDN prototype. Furthermore, considering two time steps (as Laroche does in the appendix to [61]) is also not sufficient to guarantee BIBO stability.

Now, by setting $\xi_{0}, \xi_{1}=1$, i.e., using the proposed Schroeder structures from this article, we obtain $\|\boldsymbol{A}[n]\|=\|\boldsymbol{A}[n] \boldsymbol{A}[n+1]\|=1, \forall n$. So the structure is now marginally stable, as an FDN prototype should be, under these particular time-varying coefficients.

Looking at $\boldsymbol{A}$ instead from the perspective of unitarity, we can see that when $\xi_{0}, \xi_{1}=1$, we obtain $\boldsymbol{A}=\left[\begin{array}{cc}\boldsymbol{Q} & \mathbf{0} \\ \mathbf{0} & \boldsymbol{I}_{2}\end{array}\right] \boldsymbol{U}(\underline{\boldsymbol{G}})$, where $\underline{\boldsymbol{G}}=\operatorname{diag}\left(g_{0}, g_{1}\right)$. That is, $\boldsymbol{A}$ is the product of two unitary matrices and therefore unitary.

\subsection{Correcting a General Allpass N-FDN}

Consider a MIMO "allpass FDN" in the sense of a standard $N$-FDN augmented with Schroeder allpass filters in cascade with each of its delay lines, with diagonal matrix of allpass gains $\underline{\boldsymbol{G}}$, FDN feedback gain $\boldsymbol{Q}$, and allpass defects $\underline{\boldsymbol{\Xi}}=\operatorname{diag}\left(\xi_{0}, \xi_{1}, \ldots, \xi_{N-1}\right)$, all $\in \mathbb{R}^{N \times N}$, as shown in Fig. 14(a). As in the SISO case (SEC. 4, Fig. 11(a)) the Schroeder allpass filters are realized by the generalized blocks. Assuming that we are designing an FDN reference filter, we require that $\boldsymbol{Q}$ is unitary.

Rewriting the allpass $N$-FDN as a standard $2 N$-FDN, as shown in Fig. 14(b), yields the delay state space system

$$
\begin{aligned}
& \left.\mathbf{A}=\left[\begin{array}{cc}
\mathbf{Q} \underline{\mathbf{G}} & \mathbf{Q D}_{\underline{\mathbf{G}}} \Xi \\
\mathrm{D}_{\underline{\mathbf{G}}} \Xi^{-1} & -\underline{\mathbf{G}}
\end{array}\right], \mathbf{B}=\left[\begin{array}{l}
1 \\
\mathbf{0}
\end{array}\right], \mathbf{w}=\Delta^{\left[\mathbf{m} \mathbf{m}^{\prime}\right.}\right]^{\top} \mathbf{u}, \\
& \mathbf{C}=\left[\begin{array}{llll}
g_{0} & \mathbf{0} & \xi \mathrm{D}_{g_{0}} & \mathbf{0}
\end{array}\right], \quad \mathbf{D}=[0] .
\end{aligned}
$$

Keeping in mind the usual constraints on $\underline{\boldsymbol{G}}$ and $\|\boldsymbol{Q}\|=$ 1 , we find that a necessary and sufficient criteria for unitarity of $\boldsymbol{A}$ is $\xi_{n}=1, \forall n \in\{1,2, \ldots, N-1\}$. So, using any proposed Schroeder allpass structures from SEC. 2 (Fig. 8) for each of the $N$ Schroeder allpass filter structures used here is sufficient to make an energy-preserving reference filter, no matter how each one's gain is varied over time. Considering unilosslessness $\boldsymbol{A}$ is unilossless because it is diagonally similar to $\left[\begin{array}{ll}\boldsymbol{Q} & \mathbf{0} \\ \mathbf{0} & \boldsymbol{I}\end{array}\right] \boldsymbol{U}(\boldsymbol{G})$ by $\underline{\boldsymbol{P}}=\left[\begin{array}{ll}\boldsymbol{I} & \mathbf{0} \\ \mathbf{0} & \underline{\boldsymbol{\Xi}}^{-1}\end{array}\right]$.

Contracting the constraint $\|\boldsymbol{Q}\|=1$ to $\|\boldsymbol{Q}\|<1$, we can make similar comments as in the SISO case (SEC. 4). In this case we conjecture that stability can be proven by considering $H=\max _{n}\left(m_{n}+m_{n}^{\prime}\right), n \in\{0,1, \ldots, N-1\}$ time steps, i.e., proving $\left\|\prod_{\eta=0}^{\eta=H} \boldsymbol{A}[n+\eta]\right\|<1$. Tests have shown us that setting $\xi_{n}=1, n \in\{0,1, \ldots, N-1\}$ appears to be sufficient to cause this. This shows that the proposed structures create BIBO-stable allpass FDNs while classical Schroeder 
allpass filters do not. Unfortunately we do not at this time have a formal proof.

We can also make the same comment as in the SISO case (SEC. 4) about the guaranteed stability imbued by contracting $\boldsymbol{A}$ by a gain $|\nu|<1$. This will guarantee BIBO stability and is closely related to though not identical to using a contractive $\boldsymbol{Q}$ in an allpass FDN. And, again, this would cause a certain disruption to the allpass response of each Schroeder allpass filter.

\section{CONCLUSION}

In this article we have presented novel generalizations of the (SISO) Schroeder allpass filter and (MIMO) Gerzon allpass reverberator, which, unlike the classic versions, are energy-preserving under arbitrary time-varying coefficients. These algorithms should be useful in artificial reverberation designs and audio effects like choruses and phasers, which involve time-varying allpass filters.

For the (SISO) Schroeder allpass filters we have also reviewed various properties of the LTI versions, derived a few new ones, and shown several strategies for saving multipliers in cascaded and nested structures. As with the classic Gerzon design our proposed MIMO systems are allpass by construction, although we have extended the classical design space significantly to include systems parameterized by any contractive feedback matrix $\boldsymbol{G}$, and they now enjoy good time-varying properties like the Schroeder allpass filters.

As a case study, we have examined 1-FDNs and $N$-FDNs whose delay lines have been augmented with cascaded Schroeder allpass filters. By redrawing these as standard 2-FDNs and $2 N$-FDNs we showed that the proposed structures improve on the classic design by rendering the prototype structure's feedback matrix unitary. Introducing some losses, we find that these structures are BIBO stable under time-varying coefficients, though a formal proof is left to future work.

\section{ACKNOWLEDGMENT}

Thank you to Jean-Marc Jot for helpful comments on the manuscript. Thank you to Fernando Lopez-Lezcano, Chris Chafe, and Bill Schottstaedt for their help tracking down information about KipREV.

\section{REFERENCES}

[1] M. R. Schroeder and B. F. Logan, "Colorless' Artificial Reverberation," IRE Trans. Audio, vol. AU9, no. 6, pp. 209-214 (1961 Nov./Dec.). https://doi.org/ 10.1109/TAU.1961.1166351.

[2] M. R. Schroeder, "Natural Sounding Artificial Reverberation," J. Audio Eng. Soc., vol. 10, no. 3, pp. 219-223 (1962 Jul.).

[3] J.-M. Jot and A. Chaigne, "Digital Delay Networks for Designing Artificial Reverberators," presented at the 90th Convention of the Audio Engineering Society (1991 Feb.), paper 3030 .
[4] J. A. Moorer, "About This Reverberation Business," Comput. Music J., vol. 3, no. 2, pp. 13-28 (1979 Jun.). https://doi.org/10.2307/3680280.

[5] R. Väänänen, V. Välimäki, J. Huopaniemi, and M. Karjalainen, "Efficient and Parametric Reverberator for Room Acoustics Modeling," in Proceedings of the International Computer Music Conference (ICMC'97), pp. 200203 (Thessaloniki, Greece) (1997 Sep.).

[6] R. Väänänen, Efficient Modeling and Simulation of Room Reverberation, Master's thesis, Helsinki University of Technology, Espoo, Finland (1997 Jun.).

[7] L. Dahl and J.-M. Jot, "A Reverberator Based on Absorbent All-Pass Filters," in Proceedings of the COST G6 Conference on Digital Audio Effects (DAFX-00) (Verona, Italy) (2000 Dec.).

[8] S. J. Schlecht and E. A. P. Habets, "Time-Varying Feedback Matrices in Feedback Delay Networks and Their Application in Artificial Reverberation," J. Acoust. Soc. Am., vol. 138, no. 3, pp. 1389-1398 (2015 Sep.). https://doi.org/10.1121/1.4928394.

[9] M. A. Gerzon, "Unitary (Energy-Preserving) Multichannel Networks With Feedback," Electron. Lett., vol. 12, no. 11, pp. 278-279 (1976 May). https://doi.org/10.1049/el:19760215.

[10] J. O. Smith III, Physical Audio Signal Processing for Virtual Musical Instruments and Digital Audio Effects (W3K Publishing, Denver, CO 2010).

[11] W. G. Gardner, "Reverberation Algorithms," in M. Kahrs and K. Brandenburg (Eds.), Applications of Digital Signal Processing to Audio and Acoustics, pp. 85-131 (Springer, New York, NY, 2002). https://doi.org/10.1007/ 0-306-47042-X_3.

[12] V. Kot, "Digital Sound Effects Echo and Reverb Based on Non-Exponentially Decaying Comb Filter," in Proceedings of the 14th European Signal Processing Conference (Florence, Italy) (2006 Sep.).

[13] D. H. Lee, T. H. Kim, and J. S. Park, "Design of a Digital Artificial Reverberation System Using a Dual AllPass Filter," J. Audio Eng. Soc., vol. 57, no. 3, pp. 121-130 (2009 Mar.).

[14] M. R. Bai and H. Chen, "Comparative Study of Optimal Design Strategies of Reverberators," Open Acoust. J., vol. 2, pp. 31-44 (2009). https://doi.org/10.2174/1874837600902010031.

[15] M. R. Bai and G. Bai, "Optimal Design and Synthesis of Reverberators With a Fuzzy User Interface for Spatial Audio," J. Audio Eng. Soc., vol. 53, no. 9, pp. 812825 (2005 Sep.).

[16] J. H. Ahn and R. Dudas, "Musical Applications of Nested Comb Filters for Inharmonic Resonator Effects," in Proceedings of the 39th International Computer Music Conference (ICMC), pp. 226-231 (Perth, Australia) (2013 Aug.).

[17] S. Bilbao, "Time-Varying Generalizations of AllPass Filters," IEEE Signal Process. Lett., vol. 12, no. 5, pp. 376-379 (2005 May). https://doi.org/10.1109/lsp.2005. 845602.

[18] J. Pakarinen, M. Karjalainen, V. Välimäki, and S. Bilbao, "Energy Behavior in Time-Varying Fractional 
Delay Filters for Physical Modeling Synthesis of $\mathrm{Mu}-$ sical Instruments," in Proceedings of the IEEE International Conference on Acoustics, Speech, and Signal Processing (ICASSP), pp. 1-4 (Philadelphia, PA) (2005 Mar.). https://doi.org/10.1109/ICASSP.2005.1415631.

[19] J. Pekonen, "Coefficient-Modulated First-Order Allpass Filter as Distortion Effect," in Proceedings of the 11th International Conference on Digital Audio Effects (DAFx-08), pp. 83-87 (Espoo, Finland) (2008 Sep.).

[20] V. Välimäki, J. S. Abel, and J. O. Smith, "Spectral Delay Filters," J. Audio Eng. Soc., vol. 57, no. 7/8, pp. 521-531 (2009 Jul.).

[21] J. Pekonen, V. Välimäki, J. S. Abel, and J. O. Smith, "Spectral Delay Filters With Feedback and Time-Varying Coefficients," in Proceedings of the 12th International Conference on Digital Audio Effects (DAFx-09), pp. 157-164 (Como, Italy) (2009 Sep.).

[22] J. Kleimola, J. Pekonen, H. Penttinen, V. Välimäki, and J. S. Abel, "Sound Synthesis Using an Allpass Filter Chain With Audio-Rate Coefficient Modulation," in Proceedings of the 12th International Conference on Digital Audio Effects (DAFx-09), pp. 305-312 (Como, Italy) (2009 Sep.).

[23] J. Kleimola, Nonlinear Abstract Sound Synthesis Algorithms, Ph.D. thesis, Aalto University, Espoo, Finland (2013).

[24] J. Timoney, J. Pekonen, V. Lazzarini, and V. Välimäki, "Dynamic Signal Phase Distortion Using Coefficient-Modulated Allpass Filters," J. Audio Eng. Soc., vol. 62, no. 9, pp. 596-610 (2014 Sep.). https://doi.org/10.17743/jaes.2014.0033.

[25] G. Surges, T. Smyth, and M. Puckette, "Generative Audio Systems Using Power-Preserving All-Pass Filters," Comput. Music J., vol. 40, no. 1, pp. 54-69 (2016 Spring). https://doi.org/10.1162/COMJ_a_00344.

[26] J. O. Smith III, “A New Approach to Digital Reverberation Using Closed Waveguide Networks," in Proceedings of the International Computer Music Conference, pp. 47-53 (Burnaby, Canada) (1985).

[27] T. Lokki and J. Hiipakka, "A Time-Variant Reverberation Algorithm for Reverberation Enhancement Systems," in Proceedings of the COST G-6 Conference on Digital Audio Effects (DAFX-01) (Limerick, Ireland) (2001 Dec.).

[28] B. A. Blesser, "An Interdisciplinary Synthesis of Reverberation Viewpoints," J. Audio Eng. Soc., vol. 49, no. 10, pp. 867-903 (2001 Oct.).

[29] T. Choi, Y.-C. Park, and D.-H. Youn, "Design of Time-Varying Reverberators for Low Memory Applications," IEICE Trans. Inf. Syst., vol. E91.D, no. 2, pp. 379-382 (2008 Feb.). https://doi.org/10.1093/ietisy/ e91-d.2.379.

[30] S. Schlecht and E. A. P. Habets, "Reverberation Enhancement Systems With Time-Varying Mixing Matrices," in Proceedings of the 59th International Conference: Sound Reinforcement Engineering and Technology (Montreal, Canada) (2015 Jul.), paper 3-2.

[31] K. J. Werner, "Energy-Preserving Time-Varying Schroeder Allpass Filters," in Proceedings of the 23rd International Conference on Digital Audio Effects (DAFx-20), pp. 242-249 (Vienna, Austria) (2020 Sep.).

[32] M. Gerzon, "Synthetic Stereo Reverberation: Part One," Studio Sound, vol. 13, no. 12, pp. 632-635 (1971 Dec.).

[33] M. Gerzon, "Synthetic Stereo Reverberation: Part Two," Studio Sound, vol. 14, no. 1, pp. 24-28 (1972 Jan.).

[34] S. J. Schlecht, "Allpass Feedback Delay Networks," IEEE Trans. Signal Process., vol. 69, pp. 1028-1038 (2021 Jan.). https://doi.org/10.1109/TSP.2021.3053507.

[35] S. J. Schlecht, "Frequency-Dependent Schroeder Allpass Filters," Appl. Sci., vol. 10, no. 1 (2020). https://doi.org/10.3390/app10010187.

[36] E. De Sena, H. Hacıhabiboğlu, Z. Cvetković, and J. O. Smith, "Efficient Synthesis of Room Acoustics via Scattering Delay Networks," IEEE/ACM Trans. Audio Speech Lang. Process., vol. 23, no. 9, pp. 1478-1492 (2015 Sep.). https://doi.org/10.1109/TASLP.2015.2438547.

[37] B. Alary, A. Politis, S. Schlecht, and V. Välimäki, "Directional Feedback Delay Network," J. Audio Eng. Soc., vol. 67, no. 10, pp. 752-762 (2019 Oct.). https://doi.org/10.17743/jaes.2019.0026.

[38] S. J. Schlecht and E. A. P. Habets, "Dense Reverberation With Delay Feedback Matrices," in Proceedings of the IEEE Workshop on Applications of Signal Processing to Audio and Acoustics (WASPAA) (New Paltz, NY) (2019 Oct.). https://doi.org/10.1109/WASPAA.2019. 8937284.

[39] V. Välimäki, H.-M. Lehtonen, and M. Takanen, "A Perceptual Study on Velvet Noise and Its Variants at Different Pulse Densities," IEEE Trans. Audio Speech Lang. Process., vol. 21, no. 7, pp. 1481-1488 (2013 Jul.). https://doi.org/10.1109/TASL.2013.2255281.

[40] K. J. Werner, "Generalizations of Velvet Noise and Their Use in 1-Bit Music," in Proceedings of the 22nd International Conference on Digital Audio Effects (DAFx19), pp. 25-32 (Birmingham, UK) (2019 Sep.).

[41] J. Fagerström, B. Alary, S. J. Schlecht, and V. Välimäki, "Velvet-Noise Feedback Delay Network," in Proceedings of the 23rd International Conference on Digital Audio Effects (DAFx-20), pp. 219-226 (Vienna, Austria) $(2020$ Sep.).

[42] O. Das, J. S. Abel, and E. K. Canfield-Dafilou, "Delay Network Architectures for Room and Coupled Space Modeling," in Proceedings of the 23rd International Conference on Digital Audio Effects (DAFx-20), pp. 234-241 (Vienna, Austria) (2020 Sep.).

[43] N. Meyer-Kahlen, S. J. Schlecht, and T. Lokki, "Fade-In Control for Feedback Delay Networks," in Proceedings of the 23rd International Conference on Digital Audio Effects (DAFx-20), pp. 227-233 (Vienna, Austria) (2020 Sep.).

[44] J. Dattorro, "Effect Design, Part I: Reverberator and Other Filters," J. Audio Eng. Soc., vol. 45, no. 9, pp. 660684 (1997 Sep.).

[45] J. M. Chowning, J. M. Grey, J. A. Moorer, and L. Rush, "Computer Simulation of Music Instrument Tones 
in Reverberant Environments,” Rep. STAN-M-1 (Stanford University, Stanford, CA, 1974 Jun.).

[46] B. Sz.-Nagy, C. Foias, H. Bercovici, and L. Kérchy, Harmonic Analysis of Operators on Hilbert Space, 2nd ed. (Springer, New York, NY, 2010). https://doi.org/10.1007/978-1-4419-6094-8.

[47] P. P. Vaidyanathan, Multirate Systems and Filter Banks (Prentice Hall, Hoboken, NJ, 1993).

[48] P. P. Vaidyanathan and S. K. Mitra, "Low Passband Sensitivity Digital Filters: A Generalized Viewpoint and Synthesis Procedures," Proc. IEEE, vol. 72, no. 4, pp. 404-423 (1984 Apr.). https://doi.org/10.1109/PROC.1984. 12878.

[49] J.-M. Jot, Étude Réalisation d'un Spatialisateur de Sons par Modèles Physiques et Perceptifs, Ph.D. thesis, Télécom Paris, Paris, France (1992).

[50] D. Rocchesso and J. O. Smith, "Circulant and Elliptic Feedback Delay Networks for Artificial Reverberation," IEEE Trans. Speech Audio Process., vol. 5, no. 1, pp. 51-63 (1997 Jan.). https://doi.org/10.1109/89.554269.

[51] S. J. Schlecht, "FDNTB: The Feedback Delay Network Toolbox," in Proceedings of the 23rd International Conference on Digital Audio Effects (DAFx-20), pp. 211218 (Vienna, Austria) (2020 Sep.).

[52] J. L. Kelly and C. C. Lochbaum, "Speech Synthesis," in Proceedings of the 4th International Congress on Acoustics, pp. 1-4 (Copenhagen, Denmark) (1962).

[53] S. J. Schlecht and E. A. P. Habets, "On Lossless Feedback Delay Networks," IEEE Trans. Signal Process., vol. 65, no. 6, pp. 1554-1564 (2017 Mar.). https://doi.org/ 10.1109/TSP.2016.2637323.

[54] T. S. Stilson, Efficiently-Variable Non-Oversampled Algorithms in Virtual-Analog Music Synthesis-A RootLocus Perspective, Ph.D. thesis, Stanford University, Stanford, CA (2006 Jun.).

[55] J. A. Moorer, "Signal Processing Aspects of Computer Music: A Survey," Proc. IEEE, vol. 65, no. 8, pp. 1108-1137 (1977 Aug.). https://doi.org/10.1109/ PROC.1977.10660.

[56] P. R. Samson, "A General-Purpose Digital Synthesizer,” J. Audio Eng. Soc., vol. 28, no. 3, pp. 106-113 (1980 Mar.).

[57] S. Mitra and K. Hirano, "Digital All-Pass Networks," IEEE Trans. Circuits Syst., vol. 21, no. 5, pp. 688-700 (1974 Sep.). https://doi.org/10.1109/TCS.1974. 1083908.

[58] D. C. Massie, "An Engineering Study of the Four-Multiply Normalized Ladder Filter," J. Audio Eng. Soc., vol. 41, no. 7/8, pp. 564-582 (1993 Jul.).

[59] F. E. J. Girling and E. F. Good, "The Leapfrog or Active-Ladder Synthesis," Wireless World, vol. 76, no. 1417, pp. 341-345 (1970).

[60] S. J. Schlecht and E. A. P. Habets, "Connections Between Parallel and Serial Combinations of Comb Filters and Feedback Delay Networks," in Proceedings of the International Workshop on Acoustic Signal Enhancement (IWAENC) (Aachen, Germany) (2012 Sep.).
[61] J. Laroche, "On the Stability of Time-Varying Recursive Filters," J. Audio Eng. Soc., vol. 55, no. 6, pp. 460-471 (2007 Jun.).

[62] M. A. Poletti, "A Unitary Reverberator for Reduced Colouration in Assisted Reverberation Systems," in Proceedings of the Symposium on Active Control Sound Vibration, pp. 1223-1232 (Newport Beach, CA) (1995 July).

[63] P. Diţă, "Parameterisation of Unitary Matrices," $J$. Phys. A Math. Gen., vol. 15, pp. 3465-3473 (1982).

[64] D. Griesinger, "Practical Processors and Programs for Digital Reverberation," in Proceedings of the 7th International Conference: Audio in Digital Times, pp. 187-195 (Toronto, Canada) (1989 May), paper 7-027.

[65] J. Parker and V. Välimäki, "Linear Dynamic Range Reduction of Musical Audio Using an Allpass Filter Chain," IEEE Signal Process. Lett., vol. 20, no. 7, pp. 669-672 (2013 Jul.). https://doi.org/10.1109/LSP.2013. 2263136.

[66] B. Holm-Rasmussen, H.-M. Lehtonen, and V. Välimäki, "A New Reverberator Based on Variable Sparsity Convolution," in Proceedings of the 16th International Conference on Digital Audio Effects (DAFx-13), pp. 219226 (Maynooth, Ireland) (2013 Sep.).

[67] P. R. Cook and G. P. Scavone, "PRCRev Class Reference," https://ccrma.stanford.edu/software/stk/ classstk_1_1PRCRev.html (accessed Dec. 22, 2020).

[68] B. Schottstaedt, "CLM," https://ccrma.stanford. edu/software/snd/snd/clm.html. (accessed Dec. 22, 2020).

[69] C. W. Sheeline, "An Investigation of the Effects of Direct and Reverberant Signal Interactions on Auditory Distance Perception," Report No. STAN-M-13 (Stanford University, Stanford, CA, 1982 Nov.).

[70] K. Barr, "Audio Effects: Reverberation," http://www.spinsemi.com/knowledge_base/effects.html\# Reverberation (accessed Dec. 22, 2020).

[71] S. Costello, "RIP Keith Barr," https://valhalladsp. wordpress.com/2010/08/25/rip-keith-barr/ (accessed Dec. 22, 2020).

[72] V. Välimäki, B. Holm-Rasmussen, B. Alary, and H.-M. Lehtonen, "Late Reverberation Synthesis Using Filtered Velvet Noise," Appl. Sci., vol. 7, no. 5 (2017). https://doi.org/10.3390/app7050483.

[73] D. Griesinger, "Impulse Response Measurements Using All-Pass Deconvolution," in Proceedings of the 11th International Conference: Test \& Measurement, pp. 308321 (Portland, OR) (1992 May), paper 11-035.

[74] S. Cecchi, A. Carini, and S. Spors, "Room Response Equalization-A Review," Appl. Sci., vol. 8, no. 1 (2018). https://doi.org/10.3390/app8010016.

[75] H. Kuttruff, Room Acoustics, 2nd ed. (Elsevier, Amsterdam, Netherlands, 1979).

[76] T. I. Laakso and V. Välimäki, "Energy-Based Effective Length of the Impulse Response of a Recursive Filter," in Proceedings of the IEEE International Conference on Acoustics, Speech and Signal Processing (ICASSP), pp. 1253-1256 (Seattle, WA) (1998 May). https://doi.org/10.1109/ICASSP.1998.681672.

[77] T. I. Laakso and V. Välimäki, "Energy-Based Effective Length of the Impulse Response of a Recursive Filter," 
IEEE Trans. Instr. Measure., vol. 48, no. 1, pp. 7-17 (1999 Feb.). https://doi.org/10.1109/19.755042.

[78] T. Q. Nguyen, T. I. Laakso, and R. D. Koilpillai, "Eigenfilter Approach for the Design of Allpass Filters Approximating a Given Phase Response," IEEE Trans. Signal Process., vol. 42, no. 9, pp. 2257-2263 (1994 Sep.). https://doi.org/10.1109/78.317848.

\section{APPENDIX: LTI SCHROEDER ALLPASS REVIEW}

In this section, after reviewing the basic definition, we derive a number of properties of the Schroeder allpass filter in the time (A.1) and frequency (A.2) domains. The transfer function of the LTI Schroeder allpass filter with real-valued inputs and outputs is

$$
H(z)=\frac{Y(z)}{X(z)}=\rho \frac{g+z^{-M}}{1+g z^{-M}},
$$

where $g \in \mathbb{R}$ is the "allpass gain," $\rho \in \pm 1$ is the polarity, and $M \in \mathbb{Z}_{+}$is the delay-line length. For stability, $|g|<1$. This filter can be implemented by the difference equation

$$
y[n]=\rho(g x[n]+x[n-M])-g y[n-M]
$$

Values used for Schroeder allpass gains in known reverbs and related structures are shown in Table 4. In practice, certain values of $g$ are commonly used, including $g= \pm 0.7$, $1 / \phi \approx 0.618$ (the inverse of the "golden ratio" [65-67], $\varphi=(1+\sqrt{5}) / 2 \approx 1.618)$, and $1 / 2$. In practice, positive values of $g$ are used more often than negative values.

\section{A.1 Impulse Response}

Here we study the impulse response of the Schroeder allpass filter, including an energetic analysis (A.1.1) and an analysis of its effective length (A.1.2).

The impulse response, at discrete time index $n$, of the time-invariant $M$ th-order Schroeder allpass filter is

$$
h[n]=\left\{\begin{array}{ll}
0, & n<0 \text { or } n / M \notin \mathbb{Z} \\
\rho g, & n=0 \\
\rho(-g)^{\frac{n}{M}-1}\left(1-g^{2}\right), & n / M \in \mathbb{Z} \text { and } n>0
\end{array} .\right.
$$

This impulse response is only non-zero at time indices that are integer multiples of $M$. It has a single pulse of amplitude $g$ at time $n=0$ and an exponentially decaying tail starting at time $n=M$ (oscillatory for positive $g$ and unipolar for negative $g$ ). The exponentially decaying part is bounded by an envelope

$$
h_{\mathrm{env}}[n]= \pm|g|^{\frac{n}{M}-1}\left(1-g^{2}\right) .
$$

For $n \geq M$ the maximum possible value of $h_{\text {env }}[n]$ is

$$
h_{\text {env, } \max }[n]=\frac{2 M(n-M)^{\frac{n-M}{2 M}}}{(n+M)^{\frac{n+M}{2 M}}} .
$$

For $n<M$ the envelope is unbounded as $|g| \rightarrow 0$. At time $n \geq M, h_{\text {env, } \max }[n]$ is obtained by

$$
g_{\text {env, } \max }[n]= \pm \sqrt{\frac{n-M}{n+M}} .
$$

A family of impulse responses with various gains $g$ are shown in Fig. 15. In each case $h[n]$, the impulse response itself is shown with stems, $h_{\text {env }}[n]$ is shown with a solid line in the region where it is valid and a dashed line elsewhere, and $h_{\text {env, } \max }[n]$ is shown with a dotted line. For illustrative

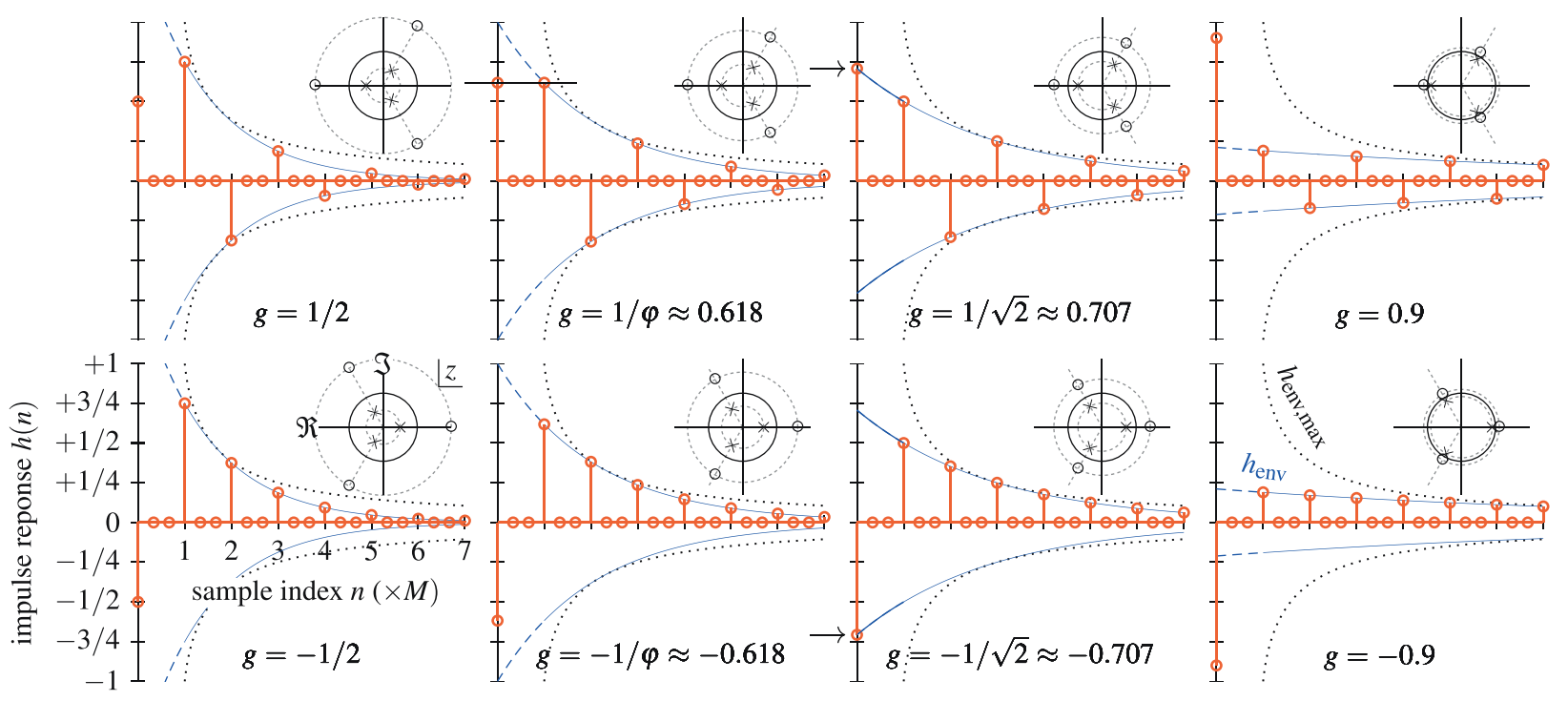

Fig. 15. Schroeder allpass filter frequency response. The center and center bottom panes show phase response $\Theta(\omega)$ and group delay $D(\omega)$ responses for $g \in\{-0.99,-0.9,-0.8, \ldots,+0.8,+0.9,+0.99\}$, for any $1 / M$ of the frequency range, $\omega \in[2 \pi m / M, 2 \pi(m+1) / M]$. The left panes show representative curves for $g= \pm 1$ and definitions of extremal group delay quantities. The way these quantities vary according to $|g|$ is shown in the lower right panel and the phase response at $\omega=2 \pi(m+1 / 4) / M$ is shown in the right pane. The top pane shows the relationship between $g$ and break frequency $\omega_{\mathrm{b}}$. The $z$-plane poles and zeros are also shown inset, where the unit circle $|z| \equiv$ 1 is shown as a solid line. 
Table 4. Summary of cascaded and nested Schroeder allpasses in reverbs and related applications. In the notation ${ }_{c}^{n} g(\times t)$ in the gains column, $c$ represents number of allpasses in cascade, $t$ represents how many times that cascade appears in the structure, and $n$ is the nested allpass filters. The last column indicates the sign convention used in each reference; the values shown are (re)written using the "positive sign convention." Reverberators below the line involve neither nestings nor cascades but are included to further illustrate common values of $g$.

\begin{tabular}{|c|c|c|c|}
\hline Author / ref & Name & Gains & \pm \\
\hline Schroeder and Logan [1] & "colorless" & $\left\{0.7,{ }_{3} 0.7,-0.7\right\}$ & - \\
\hline Cook and Scavonne [67] & PRCREV & $\left\{{ }_{2} 0.7\right\}$ & - \\
\hline Chowning [10] & JCREV & $\left\{{ }_{3} 0.7\right\}$ & + \\
\hline Chowning [10] & SATREV & $\{30.7\}$ & + \\
\hline McNabb [68] & NREV & $\left\{{ }_{3} 0.7\right\}$ & + \\
\hline Sheeline [69] & KipREV & $\left\{{ }_{4} 0.7\right\}$ & + \\
\hline Wakefield [10] & Freeverb & $\left\{{ }_{4} 0.5\right\}$ & + \\
\hline Barr $[70,71]$ & FV-1 demo & $\{20.5 \times 4\}$ & + \\
\hline Dattorro [44] & Plate & $\left\{{ }_{2} 0.75,{ }_{2} 0.625\right\}$ & \pm \\
\hline \multirow[t]{3}{*}{ Gardner [11] } & Small Room & $\left\{0.4,0.60 .3,{ }^{0.4} 0.1\right\}$ & - \\
\hline & Medium Room & $\left\{0.7,0.50 .3,{ }^{0.6} 0.3,0.5\right\}$ & - \\
\hline & Large Room & $\left\{2^{0.25} 0.5,{ }^{0.25} 0.5,{ }_{2} 0.3\right\}$ & - \\
\hline Holm-Rasmussen et al. [66] & Filt. VN Rev. & $\left\{{ }_{7}(1 / \phi) \approx 0.618\right\}$ & + \\
\hline Välimäki et al. [72] & Filt. VN Rev. & $\left\{{ }_{4} 0.7\right\}$ & + \\
\hline Fagerström et al. [41] & (Allpass) FDN & $\{70.7 \times 16,32\}$ & + \\
\hline Griesinger [73] & Schroeder AP Net. & $\{70.62\}$ & - \\
\hline Parker and Välimäki [65] & AP chain & $\left\{{ }_{3}(1 / \phi)\right\}$ & \pm \\
\hline Välimäki et al. [20] & Spectral delay filter & $\left\{{ }^{2000} g\right\},|g|<0.9$ & + \\
\hline Pakarinen et al. [18] & String model & $\left\{{ }_{126} g\right\},|g|<1$ & + \\
\hline Bai and Chen [14] & Triple nested & $\left\{\left\{^{-0.4746}-0.4623-0.5922\right\}\right.$ & - \\
\hline Bai and Bai [15] & Triple nested & $\left\{\left\{^{-0.59059}-0.88-0.77765\right\}\right.$ & - \\
\hline Ahn and Dudas [16] & Inharm. resonator & $\{ \pm 0.8 \pm 0.8\}$ & + \\
\hline Jot and Chaigne [3] & Series allpass & $\left\{{ }_{3} 1 / \phi\right\}$ & - \\
\hline Adriaensen [10] & Zita-Rev1 & $\{ \pm 0.6 \times 8\}$ & + \\
\hline Väänänen et al. [5] & Late reverb. & $\{0.5 \times 4,8\}$ & - \\
\hline Väänänen [6] & Late reverb. & $\{0.7 \times 6,8\}$ & - \\
\hline
\end{tabular}

purposes the impulse responses are shown with $\rho=1$ and $M$ $=3$; changing $\rho$ would only change the sign and changing $M$ would only change the number of zero-valued samples between pulses.

\section{A.1.1 Impulse Response Energy Analysis}

Here we analyze the energetic properties of the LTI Schroeder allpass filter's impulse response, including its total energy, its "center of gravity" in time, an energy-based length metric, and properties of particular gain values.

The energy $E_{\text {direct }}$ in the "direct path" $(n=0)$ is

$$
E_{\text {direct }}=\sum_{n=0}^{0}(h[n])^{2}=(h[0])^{2}=g^{2} .
$$

The energy $E_{\text {f.b. }}$ in the recirculating part $(n \geq 1)$ is

$$
E_{\text {f.b. }}=\sum_{n=1}^{\infty}(h[n])^{2}=\left(1-g^{2}\right)^{2} \sum_{\ell=0}^{\infty} g^{2 \ell}=1-g^{2}
$$

They sum to unity, confirming energy preservation:

$$
E_{\text {tot. }}=E_{\text {direct }}+E_{\text {f.b. }}=1 \text {. }
$$

$E_{\text {direct }}, E_{\mathrm{f} . \mathrm{b}}$, and $E_{\text {tot. }}$ are shown in Fig. 16(a) in solid lines corresponding to the right vertical axis.
The "center of gravity" of the direct path is at $n_{\text {direct }}=0$. The "center of gravity" of the recirculating part is at

$$
n_{\text {f.b. }}=\frac{\sum_{n=1}^{\infty} n(h[n])^{2}}{\sum_{n=1}^{\infty}(h[n])^{2}}=M \frac{\sum_{\ell=0}^{\infty}(\ell+1) g^{2 \ell}}{\sum_{\ell=0}^{\infty} g^{2 \ell}}=M \frac{1}{1-g^{2}}
$$

From this we can see that the "central time" [75, 76], i.e., the "center of gravity" of the entire impulse response, is always centered at $n=M$

$$
n_{\text {center }}=\frac{n_{\text {direct }} E_{\text {direct }}+n_{\text {f.b. }} E_{\text {f.b. }}}{E_{\text {direct }}+E_{\text {f.b. }}}=M .
$$

This also means that $n_{\text {f.b. }}=2 M=2 n_{\text {center }}$ when $g=$ $1 / \sqrt{2}$. $n_{\text {direct }}, n_{\text {f.b. }}$, and $n_{\text {center }}$ are shown in Fig. 16 (a) in dashed lines corresponding to the left vertical axis.

We can identify a few interesting critical values of $g$, which are commonly used in practice. To equate the energy in the direct path and the first reflection, we solve $|g|$ $=\left(1-g^{2}\right)$ by $g= \pm 2 /(1+\sqrt{5})= \pm 1 / \varphi \approx \pm 0.618$. This also minimizes the impulse response's peak value. To set an equal energy between the direct path and the entire recirculating part, we solve $g^{2}=1-g^{2}$ by $g= \pm 1 / \sqrt{2} \approx 0.707$. This also establishes a constant decay rate throughout the impulse response (solving $g /\left(1-g^{2}\right)=1 / g$ ), i.e., it aligns the pulse at $n=0$ with the exponential decay of the rest of the impulse response. 


\section{A.1.2 Impulse Response Effective Length}

Although the energy of the Schroeder allpass filter's impulse response always has its center of gravity at $n=M$, the decay time still varies. Although IIR filters ring forever we can calculate the energy-based effective length (EL) $[76,77]$ : the time it takes for a certain percentage $P$ of the impulse response's energy to pass. The EL $n_{P}$ is the smallest non-negative integer that sets the accumulated energy $E_{A}$ higher than $P$ percent of the total energy

$$
E_{A}\left[n_{P}\right]=\sum_{n=0}^{n_{P}}(h[n])^{2} \geq E_{P}=\frac{P}{100} E .
$$

Adapting the method of $[76,77]$, we find the accumulated energy $E_{A}[n]$ and EL $n_{P}$ of a Schroeder allpass filter as

$$
\begin{aligned}
& E_{A}[n]=g^{2}\left(1+\frac{(g+1 / g)^{2}\left(1+g^{2\lfloor n / M\rfloor}\right)}{1-g^{2}}\right) \\
& n_{P}=M\left\lceil\frac{\log (1-P / 100)-\log \left(1-g^{2}\right)}{\log \left(g^{2}\right)}\right\rceil,
\end{aligned}
$$

where $\lfloor\cdot\rfloor$ and $\lceil\cdot\rceil$ are the floor resp. ceiling operators. The value of $P$ that maximizes $n_{P}^{\prime}\left(n_{P}\right.$, without the ceiling operation) for a certain $g$ is

$$
P_{\max }(g)=-100\left(\mathrm{e}^{\frac{\left(g^{2}-1\right) \log \left(1-g^{2}\right)-g^{2} \log \left(g^{2}\right)}{g^{2}-1}}-1\right),
$$

which is realized for

$$
n_{P, \max }(g)=M \frac{g^{2}}{1-g^{2}} .
$$

To find the value of $g$ given a certain $n_{P, \max }$, we have

$$
g= \pm \sqrt{\frac{n_{P, \max } / M}{1+n_{P, \max } / M}} .
$$

$n_{P}$ for various $P$ and $n_{P, \max }$ are shown in Fig. 16(b), where the traces all have slight vertical offsets for legibility. Notice that we cannot really assign one particular EL to a Schroeder allpass filter with a particular gain $g$-the length always depends on the setting of $P$. Similarly the value of $g$ that maximizes $n_{P}$ also depends on $P$.

\section{A.2 Frequency Response}

In this section we give the important frequency-domain properties of an $M$ th-order Schroeder allpass filter's magnitude response (A.2.1), phase response (A.2.2), group delay (A.2.3), and poles and zeros (A.2.4).

\section{A.2.1 Magnitude Response}

Evaluating the transfer function (33) at $z=\mathrm{e}^{-\mathrm{j} \omega}$

$H\left(\mathrm{e}^{-\mathrm{j} \omega}\right)=\rho \frac{g+\mathrm{e}^{-\mathrm{j} \omega M}}{1+g \mathrm{e}^{-\mathrm{j} \omega M}}=\frac{\rho \mathrm{e}^{-\mathrm{j} \omega M}\left(1+g \mathrm{e}^{+\mathrm{j} \omega M}\right)}{1+g \mathrm{e}^{-\mathrm{j} \omega M}}$

and noting that $\left|\rho \mathrm{e}^{-\mathrm{j} \omega M}\right|=1$ and $\left|1+g \mathrm{e}^{+\mathrm{j} \omega M}\right|=\left|1+g \mathrm{e}^{-\mathrm{j} \omega M}\right|$ verifies that Schroeder allpass filters have a flat unit magnitude response:

$$
\left|H\left(\mathrm{e}^{\mathrm{j} \omega}\right)\right|=1 \Longleftrightarrow\left|X\left(\mathrm{e}^{\mathrm{j} \omega}\right)\right|=\left|Y\left(\mathrm{e}^{\mathrm{j} \omega}\right)\right|, \forall \omega \in \mathbb{R} .
$$

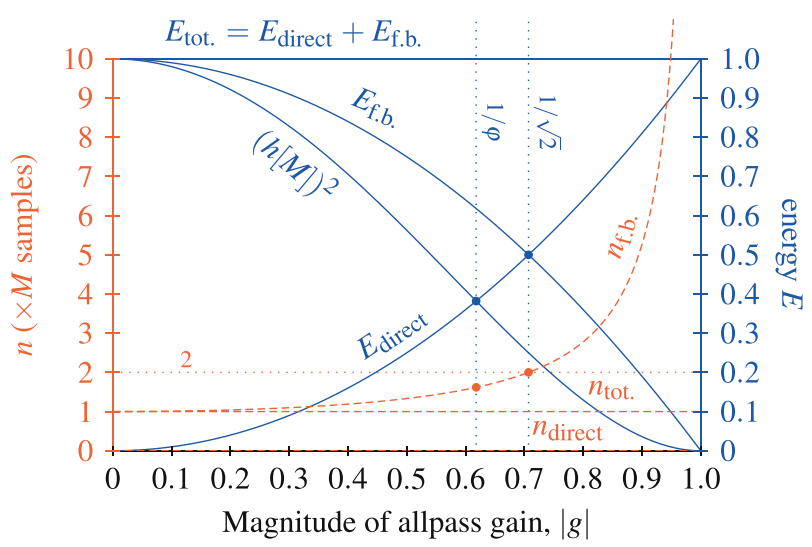

(a) Energy.

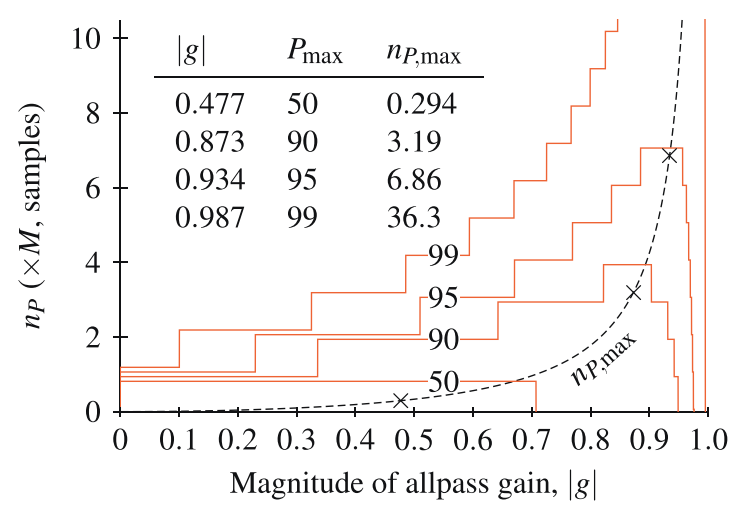

(b) Effective length.

Fig. 16. Schroeder allpass filter energy analysis.

According to the discrete-time Parseval's theorem for any signal $s[n]$ and its transform $S(\omega)$,

$$
\sum_{n=-\infty}^{\infty}|s[n]|^{2}=\frac{1}{2 \pi} \int_{-\pi}^{\pi}|S(\omega)|^{2} \mathrm{~d} \omega,
$$

(51) implies, in the time-domain [10, p. 74; 17]:

$$
\|y[\cdot]\|=\|x[\cdot]\|, \text { for any input sequence } x[n] .
$$

This is equivalent to what we found in (41). Since the filter output's energy is equal to the filter input's energy, we say that it is "energy-preserving."

The property (53) can be considered for time-varying systems as well. An energy-preserving time-varying allpass filter should satisfy (53) for a time-varying gain, and (51)(53) for a non-time-varying gain (LTI case).

\section{A.2.2 Phase Response}

The phase response $\Theta(\omega)=\angle H\left(\mathrm{e}^{\mathrm{j} \omega}\right)$ is [78]

$$
\Theta(\omega)=2 \arctan \left(\frac{g \sin (\omega M)}{1+g \cos (\omega M)}\right)-\omega M+\pi \frac{\rho+1}{2} .
$$

For $g \neq 0$, the phase response has inflection points $\left(\frac{\mathrm{d}^{2}}{\mathrm{~d} \omega^{2}} \Theta(\omega)=0\right)$ at $\omega=\pi m / M, m \in \mathbb{Z}$. It always trends downward, i.e., $\frac{d}{d \omega} \Theta(\omega)<0, \omega \in \mathbb{R}$. Swapping the polarity of $\rho$ only adds or removes an offset of $\pi$. 
The frequency at which the phase response falls to $\pi / 2$ lower than its dc response is called the "break" frequency $\omega_{b}[10]$, i.e., $\Theta\left(\omega_{b}\right)=\Theta(0)-\pi / 2$, and depends on $g$ by

$$
\omega_{\mathrm{b}}=-2 \arctan \left(\frac{1}{g}\right)-\frac{\pi}{2} \text {. }
$$
is

The gain $g_{\mathrm{b}}$ that sets the break to a certain frequency $\omega$

$$
g_{\mathrm{b}}(\omega)=-\cot \left(\frac{\omega}{2}+\frac{\pi}{4}\right) .
$$

In Fig. 17 two particular cases of the phase response for illustrative values of $g= \pm 0.7$ are shown in the middle left, the phase response itself is shown in the center, and the way that the phase response at the halfway frequencies depends on $g$ is shown in the middle right.

\section{A.2.3 Group Delay}

The group delay $D(\omega)=-\frac{\mathrm{d}}{\mathrm{d} \omega} \Theta(\Omega)$ is

$$
D(\omega)=\frac{1-g^{2}}{1+g^{2}+2 g \cos (\omega M)} M,
$$

and is shown in Fig. 17. The group delay is positive for all frequencies, i.e., $D(\omega)>0 . D(\omega)$ is entirely independent of $\rho$. For the case $g=0$, where the filter reduces to a delay line, $D(\omega)=M$.

Again, as long as $g \neq 0$, the group delay also has inflection points $\left(\frac{\mathrm{d}}{\mathrm{d} \omega} D(\omega)=0\right)$ at $\omega=\pi m / M, m \in \mathbb{Z}$, representing alternating minima and maxima of $D(\pi m / M)=$ $\frac{1-g^{2}}{1+(-1)^{m} 2 g+g^{2}}$. Specifically,

$$
\begin{aligned}
& D_{\text {peak }}=\max _{ \pm}\left\{\frac{1-g^{2}}{1 \pm 2 g+g^{2}}\right\}=\frac{1-g^{2}}{1+2|g|+g^{2}} \\
& D_{\text {valley }}=\min _{ \pm}\left\{\frac{1-g^{2}}{1 \pm 2 g+g^{2}}\right\}=\frac{1-g^{2}}{1-2|g|+g^{2}} .
\end{aligned}
$$

For $g>0$ (resp. $g<0$ ), $m \in \mathbb{Z}_{+, \text {even }}$ are minima (resp. maxima) of $D(\omega)$ and $m \in \mathbb{Z}_{+, \text {odd }}$ are maxima (resp. minima) of $D(\omega)$.

The maximum obtainable group delay for each $\omega$ is

$$
D_{\max }(\omega)=\frac{M}{|\sin (\omega M)|},
$$

obtained by

$$
g_{\max }(\omega)=\frac{|\sin (\omega M)|-1}{\cos (\omega M)} .
$$

Note that $g_{\max }(\omega)=g_{\mathrm{b}}(\omega)$; the value of $g$ that maximizes the group delay at a certain frequency is the same value that sets that frequency as the break frequency. This is shown at the top of Fig. 17. $D_{\max }(\pi m / M)=\infty, m \in \mathbb{Z}$ is not obtainable because, for $m \in \mathbb{Z}$, we get

$$
g_{\max }\left(\frac{\pi m}{M}\right)=(-1)^{m+1},
$$

which are disallowed due to the stability constraint $|g|<1$.
The Schroeder comb filter may also be described by the ratio $F$ between the minima and maxima of $D(\omega)$ [1]

$$
F=\frac{D_{\text {peak }}}{D_{\text {valley }}}=\frac{1+2|g|+g^{2}}{1-2|g|+g^{2}} .
$$

$F$ reaches its minimum of $F=1\left(D_{\text {peak }}=D_{\text {valley }}\right)$ when $g=0$, and $\lim _{g \rightarrow \pm 1} F=\infty\left(D_{\text {peak }} \rightarrow \infty, D_{\text {valley }} \rightarrow 0\right)$. A given ratio $F$ is obtained by setting

$$
g_{F}(F)=\left\{\begin{array}{ll}
0, & F=1 \\
\pm \frac{1-2 \sqrt{F}+F}{F-1}, & F>1
\end{array} .\right.
$$

Because $H(z)$ has real-valued coefficients, it is Hermitian, $\overline{H(z)}=H(-z)$, meaning that $\left|H\left(\mathrm{e}^{\mathrm{j} \omega}\right)\right|$ has even symmetry and $\Theta(\omega)$ has odd symmetry around $\omega=0$. On account of the delays $z^{-M},\left|H\left(\mathrm{e}^{\mathrm{j} \omega}\right)\right|$ and $\Theta(\omega)$ also have the same symmetries around $\omega=\pi m / M, m \in \mathbb{Z} . D(\omega)$ is even symmetric around $\omega=\pi m / M, m \in \mathbb{Z}$.

An interesting critical point is

$$
D_{\text {eq. }}=\frac{1-g^{2}}{1+g^{2}} M
$$

the group delay for which $D(\omega)$ is equal regardless of a sign flip of $g$, which occurs at $\omega=m+\frac{1+2 \mu}{4}, \mu \in \mathbb{Z}$.

In Fig. 17 the definitions of $D_{\text {max }}, D_{\text {peak }}$, and $D_{\text {valley }}$ are shown on the bottom left for two particular illustrative values of $g= \pm 0.7$; the group delay itself is shown in the bottom center; and the ways that $D_{\text {peak }}, D_{\text {valley }}$, and $D_{\text {eq. }}$. vary according to $|g|$ are shown on the bottom right.

\section{A.2.4 Poles and Zeros}

The poles $(z=p)$ resp. zeros $(z=q)$ of a Schroeder allpass filter [35] with a length- $M$ delay line come from solving the numerator resp. denominator of (33)

$$
z^{-M}=-g \quad \text { or } \quad z^{-M}=-1 / g
$$

which gives

$$
\begin{aligned}
& p_{m}=(-g)^{1 / M} \mathrm{e}^{\frac{2 \pi \mathrm{j}}{M} m}, m \in\{0,1, \ldots, M-1\} \\
& q_{m}=(-1 / g)^{1 / M} \mathrm{e}^{\frac{2 \pi \mathrm{j}}{M} m}, m \in\{0,1, \ldots, M-1\} .
\end{aligned}
$$

Alternatively these poles and zeros can be represented by their magnitudes and frequencies as

$$
p_{m}=\left|p_{m}\right| \mathrm{e}^{-\mathrm{j} \angle p_{m}} \quad \text { and } \quad q_{m}=\left|q_{m}\right| \mathrm{e}^{-\mathrm{j} \angle q_{m}},
$$

where

$$
\left|p_{m}\right|=|g|^{1 / M} \quad \text { and } \quad\left|q_{m}\right|=|1 / g|^{1 / M}
$$

$$
\angle p_{m}=\angle q_{m}=2 \pi m / M+\alpha,
$$

with

$$
\alpha=\left\{\begin{array}{ll}
\pi(\operatorname{sgn} g+1) / 2, & M \text { odd } \\
\pi(\operatorname{sgn} g+1) / 2 M, & M \text { even }
\end{array} .\right.
$$

It is well known that $\left|p_{m}\right|=1 /\left|q_{m}\right|$ and $\angle p_{m}=\angle q_{m}$, i.e., the poles and zeros are distributed uniformly in frequency and placed reciprocally across the unit circle. 

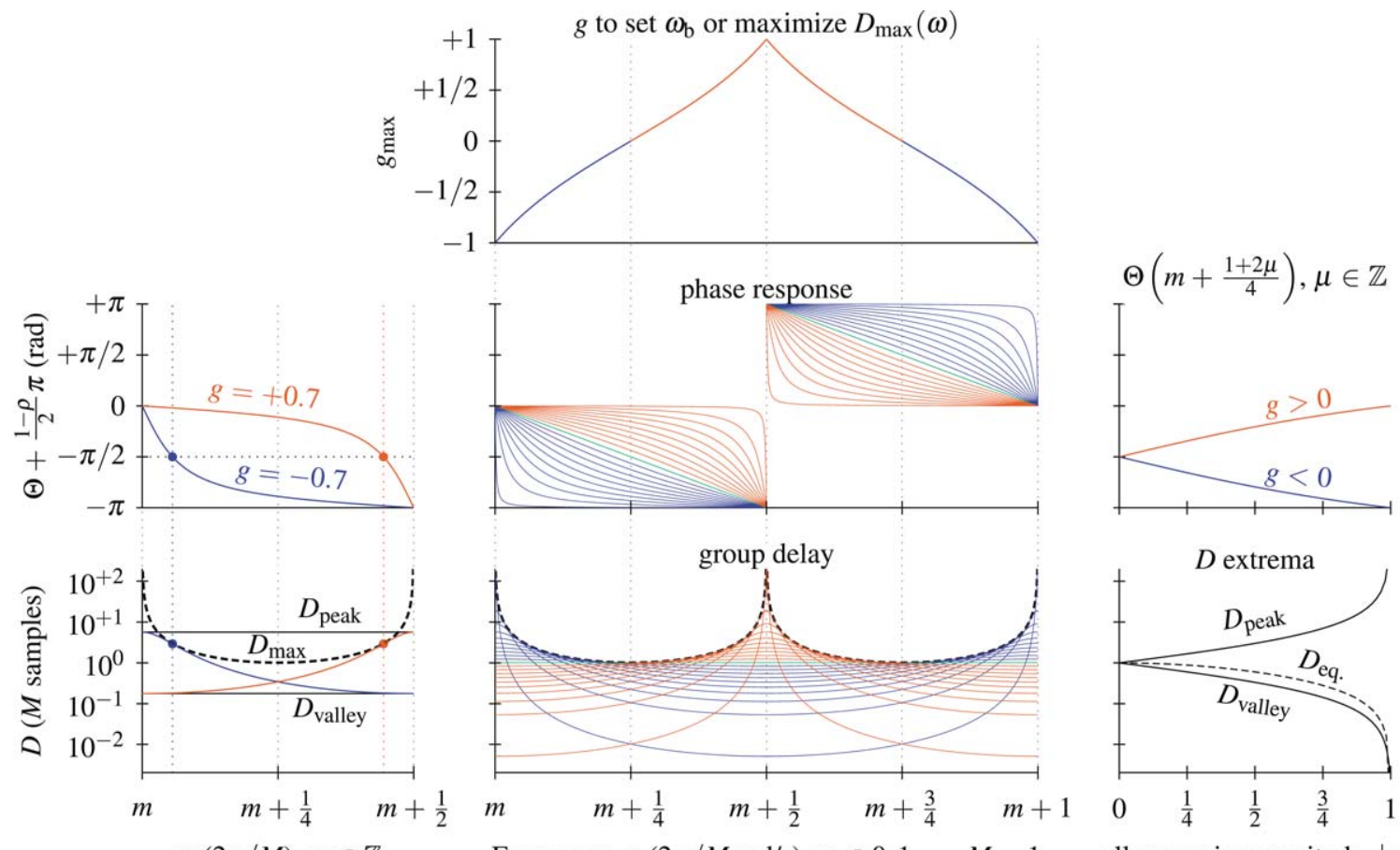

$\omega(2 \pi / M), m \in \mathbb{Z}$

Frequency $\omega(2 \pi / M \mathrm{rad} / \mathrm{s}), m \in 0,1, \ldots, M-1$

allpass gain magnitude, $|g|$

Fig. 17. Schroeder allpass filter impulse responses (in red) for various gains $g \in \pm\{1 / 2,1 / \varphi, 1 / \sqrt{2}, 0.9\}$, all with $\rho=+1$ and $M=3$. Blue solid lines represent $h_{\text {env }}$, the decaying bound on each impulse response. The black dotted line $h_{\text {env, max }}$ is the maximum value that $h_{\text {env }}$ can take for any value of $g$.

The pole magnitude expression (70) directly gives us the Schroeder allpass filter's stability criteria-to keep the poles inside of the unit circle $\left(\left|p_{m}\right|<1, m \in\{0,1, \ldots, M-\right.$ $1\}$ ) we require $|g|<1$.

Representative pole-zero plots illustrating these concepts for $g \in \pm\{1 / 2,1 / \varphi, 1 / \sqrt{2}, 0.9\}$ and $M=3$ are shown inset for each plot in Fig. 15. The unit circle is shown in a solid line and the pole and zero radii and magnitudes are shown in dashed lines. 


\section{THE AUTHORS}

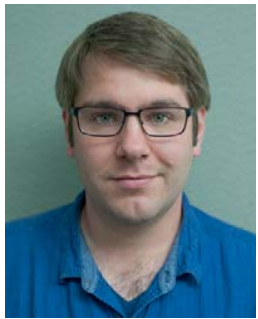

Kurt James Werner

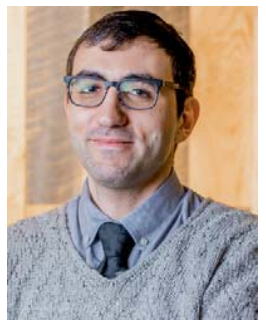

François G. Germain

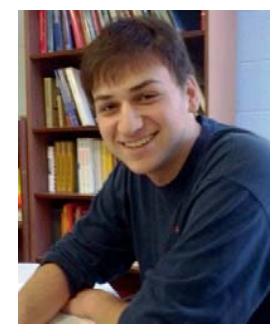

Cory S. Goldsmith
Kurt James Werner received his B.S. (2011) in General Engineering (with a secondary field in Acoustics) and B.Music in Theory and Composition, both from the University of Illinois at Urbana-Champaign (UIUC). He earned a Ph.D. (2017) in Computer-Based Music Theory and Acoustics from Stanford University's Center for Computer Research in Music and Acoustics (CCRMA). He was a Lecturer in Audio (U.K. assistant professor) at the Sonic Arts Research Centre (SARC) of Queen's University Belfast, Faculty of Arts, Humanities and Social Sciences, School of Arts, English and Languages, from 2017-2019. Since 2019 he has been a Research Engineer at iZotope, Inc. His research focuses on wave digital filters, virtual analog modeling, artificial reverb, sound synthesis, and the history of music technology. Dr. Werner is a member of IEEE, the Audio Engineering Society (AES), and the International Computer Music Association (ICMA). He was awarded best student paper by the IEEE WASPAA (2015) and second best paper by DAFx (2020). As co-author he was also awarded best paper (DAFx, 2018), best student paper (AES, 2017), best paper no. 3 (DAFx, 2016), and a best paper honorable mention (DAFx, 2015).

François Germain received a Dipl.Ing. from Ecole Poly- technique in 2010, an M.A. in Music Technology from McGill University in 2011, and an M.Sc. in Electrical Engineering and a Ph.D. in Computer-Based Music Theory and Acoustics from Stanford University, respectively in 2014 and 2019. He has been a Research Engineer at iZotope, Inc. since 2018. His research topics include signal enhancement, multichannel rendering and capture, and physical modeling of audio systems. Dr. Germain is a member of the IEEE Signal Processing Society. He was first author on articles awarded Best Student Paper at the INTERSPEECH Conference in 2013 and the 142nd Audio Engineering Society (AES) Convention in 2017. He was also awarded Best Reviewer at the International Conference on Digital Audio Effects (DAFx) and the International Society of Music Information Retrieval (ISMIR) Conference, both in 2020.

Cory Goldsmith received his B.S. (2012) in Chemistry with a minor in Mathematics from the University of Georgia. He earned a Ph.D. (2018) in Chemical Physics at JILA at the University of Colorado Boulder. His research focused on the analytical and computational modeling of the interaction of atoms and molecules with ultrafast laser pulses. Since 2019, he has been a Software Engineer at iZotope, Inc. 